\title{
Randomized allocation of oocytes to IVF or ICSI for IVF-naïve cases with unexplained infertility in an IVF-ICSI Split protocol favors ICSI to optimize live birth outcomes
}

\author{
John Lui Yovich ${ }^{1}$, 2, ${ }^{*}$, Jason Lee Conceicao ${ }^{1}$, Nicole Marjanovich ${ }^{1}$, Rachel Wicks ${ }^{1}$, Jesmine Wong ${ }^{1}$ and Peter \\ Michael Hinchliffe 1 \\ ${ }^{1}$ PIVET Medical Centre Perth, Western Australia, Australia 6007. \\ ${ }^{2}$ Department of Pharmacy and Biomedical Sciences, Curtin University Perth, Western Australia, Australia 6845.
}

GSC Biological and Pharmaceutical Sciences, 2021, 17(03), 010-037

Publication history: Received on 29 October 2021; revised on 01 December 2021; accepted on 03 December 2021

Article DOI: https://doi.org/10.30574/gscbps.2021.17.3.0346

\begin{abstract}
In assisted reproduction treatments (ART), applying the ICSI method for fertilization of oocytes rather than traditional IVF method, is regarded as controversial for two reasons, namely utility and safety. Our study examines an IVF-ICSI Split model for couples with unexplained infertility, where male factor is meticulously excluded and ART is conducted by a strict algorithm, a commitment to blastocyst culture, along with single embryo transfers and a high commitment to cryopreservation. From 242 treatment cycles, 3346 oocytes recovered (13.8 per OPU) were randomly allocated to IVF or ICSI and the fertilization rates standardized to the number of 2PNS arising from each group applying the metaphase II oocyte number identified for the ICSI group, as the denominator for both groups. The fertilization rates were significantly higher overall for ICSI (83.2\% vs 65.4\%; $<<0.0001)$, being most pronounced for women under 40 years. The resultant embryos had equivalent implantation rates in both fresh ET and frozen (FET) cycles with no significant differences in pregnancy rates, miscarriage rates or live birth outcomes indicating equivalent embryo quality. However, there were significantly higher numbers of ICSI-generated embryos cryopreserved and subsequent FET procedures showed higher live birth rates ( 21 births vs 6 births; $p<0.005)$ and potential livebirths $(214$ births vs 104 births; $\mathrm{p}<0.0001)$. No congenital fetal abnormalities were detected in any of the 199 babies delivered during the study period to December 2020, neither IVF-generated nor ICSI-generated. Whilst the data strongly favors ICSI, there were 2 women (from 26 with fertilization in one arm only) who demonstrated fertilization only in the IVF arm of the study. We conclude that the IVF-ICSI Split model should be undertaken on all IVF-naïve women with unexplained infertility to determine the appropriate fertilization mode, albeit ICSI will be safely preferred for $>90 \%$ of cases.
\end{abstract}

Keywords: Assisted reproduction treatments (ART); In vitro fertilization [IVF]; Intracytoplasmic sperm injection [ICSI]; Single embryo transfer (SET); Frozen embryo transfer (FET); Live birth productivity rate (LBPR)

\section{Introduction}

In vitro fertilization (IVF) has become a popular treatment mode for infertility due to female factors since it first success in 1978 [1-8]. With respect to infertility related to male factors, modified sperm preparation techniques were introduced from 1984 [9-11], including the addition of in-vitro adjuvant pentoxifylline [12-14], as well as micromanipulation procedures such as partial zona dissection (PZD) [15], zona drilling and later, sub-zonal insemination (SUZI) [16,17], similarly micro-insemination sperm transfer (MIST) [18] with and without pentoxifylline [17]. Finally, all the previous methods were surpassed by the introduction of intra cytoplasmic sperm injection ICSI [19] in 1992 which enabled the application of IVF to an even wider range of cases, including the use of epididymal, thereafter testicular, sperm [20] and the outcomes can benefit by the combination of pentoxifylline to overcome motility

\footnotetext{
${ }^{*}$ Corresponding author: John L Yovich

PIVET Medical Centre Perth, Western Australia Australia 6007.
} 
deficiencies in the sperm preparations prior to sperm injection [21,22]. Currently, it is estimated more than 10 million infants have been derived from the IVF \pm ICSI methodologies.

However, these historical advances in managing infertility caused some trepidation as numerous physiological processes have become sequentially bypassed. These include the molecular passport processes [23,24] underlying the selection of those approximately 20 spermatozoa which reach the oocyte-cumulus complex (OCC) within the fallopian tube for natural conception [25,26]. Even at the OCC site, several complex natural processes are bypassed when ICSI is utilized. These include hyperactivated motility [27], the acrosome reaction [28], cumulus dispersal [29], sperm-zona binding and sperm penetration followed by sperm-oolemma binding [30-31]. These processes ensue in response to oocyte-sperm activation, the molecular events being well summarized recently [32,33]. Although these processes are all by-passed by the ICSI technique, does it matter to the main desire, that of generating children for infertile couples? The subsequent processes leading to zygote formation, are completed by sperm-oocyte activation which still occurs following ICSI. There are 2 main molecular contenders for oocyte activation, namely phospholipase C-zeta and post acrosomal-WWW-P binding domain protein (PAWP) [34]. Furthermore, studies on non-human primates may also be of concern, revealing ICSI may potentially affect pronuclear orientation, cleavage patterns, and microtubule configurations (which influence zygote polarity and embryonic axes) [35-37].

Currently, there is debate around the use of ICSI for non-male factor infertility (so-called unexplained infertility) whereby the proportion of ICSI cases may rise above the standard of around 50\% [38] to levels of 80-100\%. PIVET currently applies an ICSI rate $\sim 90 \%$ justified by the demonstration of a higher number of live births. The PIVET protocols include the idea of an IVF-ICSI Split protocol for all IVF-naïve cases with unexplained infertility to avoid cases of complete fertilization failure [39,40]. Furthermore, it can define the best approach for future IVF treatments, particularly where reduced rates of fertilization may occur in one or other arm of the protocol [41-42]. A higher number of live births has recently been shown from this 3-arm policy where IVF-ICSI Split is applied for IVF-naïve cases presenting with unexplained infertility and either IVF or ICSI is undertaken in future cycles accordingly [43]. This study examines the outcomes from the IVF-ICSI Split arm over the past decade.

\section{Material and methods}

The data presented here is an observational study covering the most recent 10-year period of activity (2011-2020) in an ART facility which has an historical pioneer connection with the earliest forays into human IVF $[3,4]$. The data has been collected into the internal validated data base in real-time and analyzed retrospectively.

PIVET was established in 1981 following team leader JLY returning from his 4-year study period in London establishing an IVF facility with Professor Ian Craft across the period 1976-1980 [3-5]. Pregnancies from IVF were established in the latter half of 1981 and the first live birth occurred in July 1982 [44]. The insemination procedures were conducted by standard IVF protocols, and ICSI commenced in 1994 after a protracted period of deliberations by PIVET's Human Research Ethics Committee (HREC). ICSI was initially applied for clear cases of identifiable severe male factor, and later introduced for cases of poorly explained infertility. For this study, the following protocols have been applied:

\subsection{ART Protocols at PIVET}

PIVET's IVF procedures have been well documented over the 40 years of operation and standardized across this study period 2011-2019 [7,45]. The clinical management is undertaken according to defined FSH-dosing Algorithms, one of which is shown in Table 1 and was first published in 2012 [46] with validation of an optimized pregnancy productivity rate, and almost complete avoidance of ovarian hyperstimulation syndrome (OHSS) [47]. In almost 90\% of OPU's oocyte numbers range from 8-12 for standard cases, adapted for a long-acting FSH product [48] and adjusted for lower numbers suited to a low-cost, minimal intervention program [49]. 
Table 1 One of PIVET's FSH-dosing Algorithms enabling increments of 12.5 IU: suited for Gonal-F or biosimilars across the range of 37.5-450 IU (red sector), Puregon across the range 125-450 IU (orange sector) and Elonva in the narrow range of 200-400 IU (green sector). A separate Algorithm for Puregon enables 8.3 IU increments in the range of 41.7125 IU. Dosages are increased according to the woman's Age, diminishing AFCs and elevated BMI as well as her baseline FSH level, her smoking history, and the consideration of banking oocytes for self or donating. The Algorithm is presented as Table 1a for AFC Groups A, A+ and A++ with high AFC's $>20$ follicles; Table $1 \mathrm{~b}$ for Groups B and C with mid-range AFCs 9-19 follicles; and Table 1c for Groups D and E with low AFCs $\leq 8$ follicles. The legend for all tables is under Table $1 \mathrm{c}$

Table 1a PIVET rFSH Dosing Chart suits Gonal-F \& Biosimilars

\begin{tabular}{|c|c|c|c|c|c|c|c|c|c|c|c|c|c|c|c|c|}
\hline \multirow{2}{*}{\multicolumn{2}{|c|}{$\begin{array}{c}\text { AMH } \\
\text { AFC* }\end{array}$}} & \multicolumn{5}{|c|}{$>30 \mathrm{pmol} / \mathrm{L}$} & \multicolumn{5}{|c|}{$25-29.9 \mathrm{pmol} / \mathrm{L}$} & \multicolumn{5}{|c|}{$20-24.9 \mathrm{pmol} / \mathrm{L}$} \\
\hline & & \multicolumn{5}{|c|}{$A++(\geq 40$ follicles $)$} & \multicolumn{5}{|c|}{$A+(30-39$ follicles $)$} & \multicolumn{5}{|c|}{ A (20-29 follicles) } \\
\hline \multicolumn{2}{|c|}{$\begin{array}{l}\mathrm{BMI} \\
\mathrm{kg} / \mathrm{m}^{2}\end{array}$} & $\begin{array}{l}16- \\
17\end{array}$ & $\begin{array}{l}18- \\
19\end{array}$ & $\begin{array}{l}20- \\
21\end{array}$ & $\begin{array}{l}22- \\
29\end{array}$ & $\begin{array}{l}30- \\
35\end{array}$ & $\begin{array}{l}16- \\
17\end{array}$ & $\begin{array}{l}18- \\
19\end{array}$ & $\begin{array}{l}20- \\
21\end{array}$ & $\begin{array}{l}22- \\
29\end{array}$ & $\begin{array}{l}30- \\
35\end{array}$ & $\begin{array}{l}16- \\
17\end{array}$ & $\begin{array}{l}18- \\
19\end{array}$ & $\begin{array}{l}20- \\
21\end{array}$ & $\begin{array}{l}22- \\
29\end{array}$ & $\begin{array}{l}30- \\
35\end{array}$ \\
\hline \multirow{26}{*}{$\begin{array}{l}\text { Age } \\
\text { (yrs) }\end{array}$} & 20 & 37.5 & 37.5 & 7.5 & 50.0 & 50.0 & 50 & 50.0 & 62.5 & 62.5 & 62.5 & 75.0 & 75.0 & 75.0 & 87.5 & 87.5 \\
\hline & 21 & 37.5 & 37.5 & 37.5 & 50.0 & 50.0 & 50 & 50.0 & 62.5 & 62.5 & 62.5 & 75.0 & 75.0 & 75.0 & 87.5 & 87.5 \\
\hline & 22 & 37.5 & 50.0 & 50.0 & 50.0 & 50.0 & 62.5 & 62.5 & 62.5 & 75.0 & 75.0 & 75.0 & 87.5 & 87.5 & 87.5 & 100.0 \\
\hline & 23 & 50.0 & 50.0 & 50.0 & 62.5 & 62.5 & 62.5 & 75.0 & 75.0 & 75.0 & 87.5 & 87.5 & 87.5 & 100.0 & 100.0 & 100.0 \\
\hline & 24 & 50.0 & 50.0 & 62.5 & 62.5 & 62.5 & 75.0 & 75.0 & 75.0 & 87.5 & 87.5 & 87.5 & 100.0 & 100.0 & 100.0 & 112.5 \\
\hline & 25 & 50.0 & 62.5 & 62.5 & 62.5 & 75.0 & 75.0 & 75.0 & 87.5 & 87.5 & 87.5 & 100.0 & 100.0 & 100.0 & 112.5 & 112.5 \\
\hline & 26 & 62.5 & 62.5 & 62.5 & 75.0 & 75.0 & 75 & 87.5 & 87.5 & 87.5 & 100.0 & 100.0 & 100.0 & 112.5 & 112.5 & 112.5 \\
\hline & 27 & 62.5 & 62.5 & 75.0 & 75.0 & 75.0 & 87.5 & 87.5 & 87.5 & 100.0 & 100.0 & 100.0 & 112.5 & 112.5 & 112.5 & 125.0 \\
\hline & 28 & 62.5 & 75.0 & 75.0 & 75.0 & 87.5 & 87.5 & 87.5 & 100.0 & 100.0 & 100.0 & 112.5 & 112.5 & 112.5 & 125.0 & 125.0 \\
\hline & 29 & 75.0 & 75.0 & 75.0 & 87.5 & 87.5 & 87.5 & 100.0 & 100.0 & 100.0 & 112.5 & 112.5 & 112.5 & 125.0 & 125.0 & 125.0 \\
\hline & 30 & 75.0 & 75.0 & 75.0 & 87.5 & 87.5 & 100.0 & 100.0 & 100.0 & 112.5 & 112.5 & 112.5 & 125.0 & 125.0 & 125.0 & 137.5 \\
\hline & 31 & 75.0 & 75.0 & 87.5 & 87.5 & 100.0 & 100.0 & 100.0 & 112.5 & 112.5 & 112.5 & 125.0 & 125.0 & 125.0 & 137.5 & 137.5 \\
\hline & 32 & 87.5 & 87.5 & 87.5 & 100.0 & 100.0 & 100 & 112.5 & 112.5 & 112.5 & 125.0 & 125.0 & 125.0 & 137.5 & 137.5 & 137.5 \\
\hline & 33 & 87.5 & 87.5 & 87.5 & 100.0 & 100.0 & 100 & 112.5 & 112.5 & 112.5 & 125.0 & 125.0 & 125.0 & 137.5 & 137.5 & 150.0 \\
\hline & 34 & 87.5 & 87.5 & 100.0 & 100.0 & 112.5 & 113 & 112.5 & 112.5 & 125.0 & 125.0 & 125.0 & 137.5 & 137.5 & 150.0 & 150.0 \\
\hline & 35 & 100.0 & 100.0 & 112.5 & 112.5 & 112.5 & 125.0 & 125.0 & 125.0 & 137.5 & 137.5 & 137.5 & 150.0 & 150.0 & 162.5 & 162.5 \\
\hline & 36 & 100.0 & 100.0 & 112.5 & 112.5 & 125.0 & 125.0 & 125.0 & 137.5 & 137.5 & 137.5 & 150.0 & 150.0 & 162.5 & 162.5 & 175.0 \\
\hline & 37 & 100.0 & 100.0 & 112.5 & 112.5 & 125.0 & 125.0 & 137.5 & 137.5 & 150.0 & 150.0 & 162.5 & 162.5 & 175.0 & 175.0 & 187.5 \\
\hline & 38 & 112.5 & 112.5 & 125.0 & 125.0 & 125.0 & 125 & 137.5 & 137.5 & 150.0 & 150.0 & 162.5 & 162.5 & 175.0 & 175.0 & 187.5 \\
\hline & 39 & 112.5 & 112.5 & 125.0 & 125.0 & 137.5 & 138 & 137.5 & 150.0 & 150.0 & 162.5 & 162.5 & 175.0 & 175.0 & 187.5 & 187.5 \\
\hline & 40 & 112.5 & 112.5 & 125.0 & 137.5 & 137.5 & 138 & 150.0 & 150.0 & 150.0 & 162.5 & 162.5 & 175.0 & 187.5 & 187.5 & 200.0 \\
\hline & 41 & 125.0 & 125.0 & 137.5 & 137.5 & 150.0 & 150.0 & 150.0 & 162.5 & 162.5 & 162.5 & 175.0 & 187.5 & 187.5 & 200.0 & 200.0 \\
\hline & 42 & 125.0 & 125.0 & 137.5 & 150.0 & 150.0 & 163 & 162.5 & 175.0 & 175.0 & 187.5 & 187.5 & 200.0 & 200.0 & 212.5 & 225.0 \\
\hline & 43 & 125.0 & 137.5 & 150.0 & 150.0 & 162.5 & 163 & 175.0 & 175.0 & 187.5 & 187.5 & 200.0 & 212.5 & 212.5 & 237.5 & 250.0 \\
\hline & 44 & 137.5 & 137.5 & 150.0 & 162.5 & 175.0 & 175.0 & 187.5 & 187.5 & 200.0 & 200.0 & 212.5 & 225.0 & 225.0 & 237.5 & 250.0 \\
\hline & 45 & 150.0 & 150.0 & 162.5 & 162.5 & 175.0 & 175.0 & 187.5 & 187.5 & 200.0 & 200.0 & 212.5 & 225.0 & 225.0 & 250.0 & 250.0 \\
\hline
\end{tabular}




\begin{tabular}{|c|c|c|c|c|c|c|c|c|c|c|c|}
\hline \multicolumn{12}{|c|}{ Table 1b PIVET rFSH Dosing Chart suits Gonal-F, Puregon and Elonva } \\
\hline \multicolumn{2}{|l|}{ AMH } & \multicolumn{5}{|c|}{$15-19.9 \mathrm{pmol} / \mathrm{L}$} & \multicolumn{5}{|c|}{$10-14.9 \mathrm{pmol} / \mathrm{L}$} \\
\hline \multirow{2}{*}{\multicolumn{2}{|c|}{$\begin{array}{r}\text { AFC* } \\
\mathrm{BMI} \mathrm{kg} / \mathrm{m}^{2}\end{array}$}} & \multicolumn{5}{|c|}{ B (13-19 follicles) } & \multicolumn{5}{|c|}{ C (9-12 follicles) } \\
\hline & & $16-17$ & 18-19 & $20-21$ & $22-29$ & 30-35 & $16-17$ & $18-19$ & $20-21$ & $22-29$ & 30-35 \\
\hline \multirow[t]{26}{*}{ Age (years) } & 20 & 100.0 & 100.0 & 100.0 & 112.5 & 112.5 & 125.0 & 125.0 & 137.5 & 137.7 & 137.7 \\
\hline & 21 & 100.0 & 100.0 & 100.0 & 112.5 & 112.5 & 125.0 & 125.0 & 137.5 & 137.7 & 137.7 \\
\hline & 22 & 100.0 & 100.0 & 112.5 & 112.5 & 112.5 & 125.0 & 125.0 & 137.5 & 137.5 & 150.0 \\
\hline & 23 & 112.5 & 112.5 & 112.5 & 125.0 & 125.0 & 125.5 & 137.5 & 137.5 & 150.0 & 150.0 \\
\hline & 24 & 112.5 & 112.5 & 125.0 & 125.0 & 125.0 & 137.5 & 137.5 & 150.0 & 150.0 & 162.5 \\
\hline & 25 & 112.5 & 125.0 & 125.0 & 125.0 & 137.5 & 137.5 & 150.0 & 150.0 & 150.0 & 162.5 \\
\hline & 26 & 125.0 & 125.0 & 125.0 & 137.5 & 137.5 & 137.5 & 150.0 & 150.0 & 162.5 & 162.5 \\
\hline & 27 & 125.0 & 125.0 & 137.5 & 137.5 & 137.5 & 150.0 & 150.0 & 150.0 & 162.5 & 162.5 \\
\hline & 28 & 125.0 & 137.5 & 137.5 & 137.5 & 150.0 & 150.0 & 150.0 & 162.5 & 162.5 & 175.0 \\
\hline & 29 & 137.5 & 137.5 & 137.5 & 150.0 & 150.0 & 162.5 & 162.5 & 175.0 & 175.0 & 187.5 \\
\hline & 30 & 137.5 & 137.5 & 150.0 & 150.0 & 150.0 & 162.5 & 162.5 & 175.0 & 175.0 & 187.5 \\
\hline & 31 & 137.5 & 150.0 & 150.0 & 150.0 & 162.5 & 162.5 & 175.0 & 175.0 & 187.5 & 187.5 \\
\hline & 32 & 150.0 & 150.0 & 162.5 & 162.5 & 175.0 & 175.0 & 187.5 & 187.5 & 200.0 & 200.0 \\
\hline & 33 & 150.0 & 150.0 & 162.5 & 162.5 & 175.0 & 187.5 & 200.0 & 200.0 & 212.5 & 212.5 \\
\hline & 34 & 162.5 & 162.5 & 175.0 & 175.0 & 187.5 & 187.5 & 200.0 & 212.5 & 225.0 & 237.5 \\
\hline & 35 & 175.0 & 175.0 & 175.0 & 187.5 & 200.0 & 200.0 & 212.5 & 225.0 & 237.5 & 250.0 \\
\hline & 36 & 175.0 & 187.5 & 200.0 & 200.0 & 225.0 & 225.0 & 237.5 & 237.5 & 250.0 & 262.5 \\
\hline & 37 & 187.5 & 200.0 & 212.5 & 212.5 & 225.0 & 237.5 & 250.0 & 262.5 & 275.0 & 287.5 \\
\hline & 38 & 187.5 & 200.0 & 212.5 & 225.0 & 237.5 & 250.0 & 262.5 & 275.0 & 287.5 & 300.0 \\
\hline & 39 & 200.0 & 212.5 & 225.0 & 237.5 & 250.0 & 275.0 & 287.5 & 300.0 & 312.5 & 325.0 \\
\hline & 40 & 225.0 & 237.5 & 250.0 & 262.5 & 275.0 & 300.0 & 312.5 & 325.0 & 337.5 & 350.0 \\
\hline & 41 & 225.0 & 250.0 & 262.5 & 275.0 & 287.5 & 300.0 & 325.0 & 350.0 & 375.0 & 400.0 \\
\hline & 42 & 250.0 & 262.5 & 275.0 & 287.5 & 300.0 & 325.0 & 350.0 & 375.0 & 400.0 & 425.0 \\
\hline & 43 & 262.5 & 275.0 & 287.5 & 300.0 & 312.5 & 350.0 & 375.0 & 400.0 & 425.0 & 450.0 \\
\hline & 44 & 275.0 & 275.0 & 312.5 & 325.0 & 350.0 & 375.0 & 400.0 & 425.0 & 450.0 & 450.0 \\
\hline & 45 & 287.5 & 300.0 & 325.0 & 350.0 & 362.5 & 400.0 & 425.0 & 450.0 & 450.0 & 450.0 \\
\hline
\end{tabular}

\section{Table 1c PIVET rFSH Dosing Chart suits Gonal-F, Puregon and Elonva}

\begin{tabular}{|c|c|c|c|c|c|c|c|c|c|c|c|}
\hline \multicolumn{2}{|c|}{ AMH } & \multicolumn{5}{|c|}{$5.0-9.9 \mathrm{pmol} / \mathrm{L}$} & \multicolumn{5}{|c|}{$<5.0 \mathrm{pmol} / \mathrm{L}$} \\
\hline \multirow{2}{*}{\multicolumn{2}{|c|}{$\frac{\mathrm{AFC}^{*}}{\mathrm{BMI} \mathrm{kg} / \mathrm{m}^{2}}$}} & \multicolumn{5}{|c|}{ D (5-8 follicles) } & \multicolumn{5}{|c|}{ E $(\leq 4$ follicles $)$} \\
\hline & & $16-17$ & $18-19$ & $20-21$ & $22-29$ & $30-35$ & $16-17$ & $18-19$ & $20-21$ & $22-29$ & $30-35$ \\
\hline \multirow{8}{*}{$\begin{array}{l}\text { Age } \\
\text { (years) }\end{array}$} & 20 & 150.0 & 150.0 & 162.5 & 162.5 & 175.0 & 175.0 & 187.5 & 187.5 & 200.0 & 200.0 \\
\hline & 21 & 150.0 & 150.0 & 162.5 & 162.5 & 175.0 & 175.0 & 187.5 & 187.5 & 200.0 & 200.0 \\
\hline & 22 & 150.0 & 162.5 & 162.5 & 175.0 & 175.0 & 187.5 & 187.5 & 187.5 & 200.0 & 200.0 \\
\hline & 23 & 162.5 & 162.5 & 175.0 & 175.0 & 187.5 & 187.5 & 187.5 & 200.0 & 200.0 & 212.5 \\
\hline & 24 & 162.5 & 175.0 & 175.0 & 187.5 & 187.5 & 187.5 & 200.0 & 200.0 & 212.5 & 212.5 \\
\hline & 25 & 162.5 & 175.0 & 175.0 & 187.5 & 187.5 & 200.0 & 200.0 & 200.0 & 212.5 & 212.5 \\
\hline & 26 & 175.0 & 175.0 & 175.0 & 187.5 & 187.5 & 200.0 & 200.0 & 212.5 & 212.5 & 225.0 \\
\hline & 27 & 175.0 & 175.0 & 187.5 & 187.5 & 200.0 & 200.0 & 212.5 & 212.5 & 225.0 & 225.0 \\
\hline
\end{tabular}




\begin{tabular}{|l|l|l|l|l|l|l|l|l|l|l|l|}
\hline 28 & 175.0 & 187.5 & 187.5 & 200.0 & 200.0 & 212.5 & 212.5 & 225.0 & 225.0 & 237.5 \\
\hline 29 & 187.5 & 187.5 & 200.0 & 200.0 & 212.5 & 212.5 & 225.0 & 225.0 & 237.5 & 250.0 \\
\hline 30 & 187.5 & 200.0 & 200.0 & 212.5 & 212.5 & 225.0 & 225.0 & 237.5 & 250.0 & 262.5 \\
\hline 31 & 200.0 & 200.0 & 212.5 & 212.5 & 225.0 & 237.5 & 250.0 & 262.5 & 275.0 & 287.5 \\
\hline 32 & 212.5 & 225.0 & 237.5 & 250.0 & 262.5 & 275.0 & 287.5 & 300.0 & 312.5 & 325.0 \\
\hline 33 & 225.0 & 237.5 & 250.0 & 262.5 & 275.0 & 287.5 & 300.0 & 312.5 & 325.0 & 337.5 \\
\hline 34 & 250.0 & 262.5 & 275.0 & 287.5 & 300.0 & 325.0 & 350.0 & 375.0 & 400.0 & 425.0 \\
\hline 35 & 275.0 & 287.5 & 300.0 & 325.0 & 350.0 & 362.5 & 375.0 & 400.0 & 425.0 & 450.0 \\
\hline 36 & 275.0 & 287.5 & 300.0 & 325.0 & 350.0 & 375.0 & 400.0 & 425.0 & 450.0 & 450.0 \\
\hline 37 & 300.0 & 325.0 & 350.0 & 362.5 & 375.0 & 400.0 & 425.0 & 450.0 & 450.0 & 450.0 \\
\hline 38 & 325.0 & 350.0 & 375.0 & 400.0 & 425.0 & 450.0 & 450.0 & 450.0 & 450.0 & 450.0 \\
\hline 39 & 350.0 & 375.0 & 400.0 & 425.0 & 450.0 & 450.0 & 450.0 & 450.0 & 450.0 & 450.0 \\
\hline 40 & 375.0 & 400.0 & 425.0 & 450.0 & 450.0 & 450.0 & 450.0 & 450.0 & 450.0 & 450.0 \\
\hline 41 & 425.0 & 450.0 & 450.0 & 450.0 & 450.0 & 450.0 & 450.0 & 450.0 & 450.0 & 450.0 \\
\hline 42 & 450.0 & 450.0 & 450.0 & 450.0 & 450.0 & 450.0 & 450.0 & 450.0 & 450.0 & 450.0 \\
\hline 43 & 450.0 & 450.0 & 450.0 & 450.0 & 450.0 & 450.0 & 450.0 & 450.0 & 450.0 & 450.0 \\
\hline 44 & 450.0 & 450.0 & 450.0 & 450.0 & 450.0 & 450.0 & 450.0 & 450.0 & 450.0 & 450.0 \\
\hline & 45 & 450.0 & 450.0 & 450.0 & 450.0 & 450.0 & 450.0 & 450.0 & 450.0 & 450.0 & 450.0 \\
\hline
\end{tabular}

\begin{tabular}{|c|c|}
\hline Increased basal FSH and Smokers & Standard Dosing as above \\
\hline $\begin{array}{l}\text { Where FSH is less than } 8 \mathrm{IU} / \mathrm{L} \text {, with no } \\
\text { history of smoking, use values as shown }\end{array}$ & \\
\hline \multirow{2}{*}{$\begin{array}{l}\text { Smokers move } 2 \text { columns to the right } \\
\text { Where FSH is between } 8 \text { and } 12 \mathrm{IU} / \mathrm{L} \text {, with } \\
\text { no history of smoking, move one column to } \\
\text { the right }\end{array}$} & Aiming for $10-15$ oocytes, \\
\hline & move four columns to the right \\
\hline \multirow{2}{*}{$\begin{array}{l}\text { Smokers move two columns to the right } \\
\text { Where FSH is greater than } 12 \mathrm{IU} / \mathrm{L} \text {, move } \\
\text { two columns to the right }\end{array}$} & $\begin{array}{l}\text { Consider GnRH antagonist Trigger } \\
\text { if }>10 \text { follicles }\end{array}$ \\
\hline & e.g., Tryptorelin $100 \mu \mathrm{g} \mathrm{X} 2$ \\
\hline \multicolumn{2}{|l|}{$\begin{array}{l}\text { Smokers and non-smokers read same } \\
\text { column }\end{array}$} \\
\hline \multicolumn{2}{|l|}{$\begin{array}{l}\text { *Antral Follicle Count based on number of } \\
\text { antral follicles }<1.0 \mathrm{~cm}\end{array}$} \\
\hline \multicolumn{2}{|l|}{ Colour Scheme } \\
\hline \multirow{3}{*}{\multicolumn{2}{|c|}{$\begin{array}{l}\square \quad 25 \text { IU increments also suits Puregon } \\
\square \quad \text { Elonva. } 1 \times 100 \text { for weight }<60 \mathrm{~kg}\end{array}$}} \\
\hline & \\
\hline & \\
\hline $1 \times 150 \mu \mathrm{g}$ for weight $>60 \mathrm{~kg}$ & \\
\hline
\end{tabular}

Oocyte pick-up is conducted with a Cook single-lumen aspirating needle where follicle numbers exceed 5, and the PIVET-Cook double lumen needle where follicle numbers are $\leq 5$ to enable combined aspiration and flushing $[7,45]$. From the laboratory perspective, PIVET has increasingly applied a blastocyst culture system ( $90 \%$ of embryos) followed by a SET regimen ( $91 \%$ of cases) and commitment to cryopreservation by vitrification using the Kuwayama method [50]. Under an ANZARD initiative, the results from all participating IVF units in Australia and New Zealand can now be 
viewed [51] and reveals that PIVET's IVF program generates live birth productivity rates [52] in the highest quartile across all age groups of women. These high live birth productivity rates are achieved whilst recording multiple pregnancies in $<5 \%$ of births (being twins only) and complications such as severe ovarian hyperstimulation syndrome (OHSS) requiring hospitalization occurring in only 1/ 1000 OPU cases.

\subsection{Indications for ICSI}

The range of indications for ICSI at PIVET includes male factor causes and extends across a range of non-male factor scenarios according to the recently reported study [43].

\subsubsection{Male infertility factors}

Male infertility is diagnosed according to the semen parameters defined by the WHO laboratory manual of 2010 [53] which delineates those samples with oligozoospermia, asthenozoospermia or teratozoospermia, and combinations of these, particularly that known as OAT (oligo-astheno-teratozoospermia) syndrome. However, PIVET also includes those men with identifiable anti-spermatozoal antibodies in their semen (IgG levels $>20 \%$ ) and those with significant levels of DNA fragmentation ( $\geq 15 \%$ ) identified by the Halo test [54]. Male factor infertility also includes those cases requiring surgical sperm retrieval [20] including microsurgical epididymal sperm aspiration (MESA), percutaneous epidydimal sperm aspiration (PESA) and testicular sperm aspirations (TESA and micro-TESE) as well as Vasal Flush procedures, mostly applied for males with spinal injury [36]. Although PIVET does not apply direct testing of semen for ROS, cases of obstructive azoospermia are considered likely to have excessive ROS as an underlying reason for poor fertilizing capacity.

Clinical conditions of the male are also considered for ICSI. These include morbid obesity, the presence of clinically detectable varicocele, the presence of ejaculatory disorders, a history of maldescent of the testis, a history of epididymitis or testicular torsion, and any orchidopexy procedure. In addition, males with chronic disease especially involving chemotherapy or radiotherapy, or those with infectious disease such as HIV, Hepatitis B and Hepatitis C were also advised to use ICSI insemination. Finally, those on drug therapy which may affect fertilizing capacity such as sulphasalazine, cimetidine, allopurinol amongst others, along with males who use recreational drugs or are exposed to chemicals and heavy metals in "risky" occupations such as welding [56], are also encouraged to utilize ICSI.

\subsubsection{Female factor infertility}

Advanced female age as well as reduced ovarian reserve are inter-connected to lower antral follicle counts (AFCs) and low serum anti- Mullerian hormone (AMH). These combinations are closely associated with poor IVF prognosis [57], which includes an array of deficiencies such as reduced fertilization of oocytes and low oocyte numbers on retrieval. ICSI can at least improve oocyte fertilization, although it may not have any major influence over embryo quality or implantation potential. It does however reduce the problem of polyspermic IVF fertilization seen more frequently in oocytes from women of advanced age or those with diminished ovarian reserve [58].

It was reported by Diedrich's group that where $<4$ oocytes are re- covered, ICSI guarantees a successful treatment outcome more often than IVF and encourages the idea of milder forms of stimulation [59]. Our clinic has adopted a milder stimulation policy in recent years such that many women will now generate $<5$ oocytes where ICSI provides a greater chance of generating embryos for transfer, especially if few oocytes [60] or only a single oocyte is retrieved [61]. Finally, oocyte anomalies are also linked to advanced female age and poor prognosis cases. Zona thickening is associated with advanced maternal age and zona hardening is associated with cryopreservation, especially for immature oocytes. The consequential effect is reduced or failed fertilization [62] and this appears to be related to the degree of response to gonadotrophin stimulation. Some zona problems can be encountered leading to reduced or absent sperm binding and these can mostly be resolved by ICSI [63].

\subsubsection{Unexplained or poorly explained infertility}

Whilst large RCT studies indicate that unexplained infertility is not, by itself an indication for ICSI, the outcomes of any IVF application may reveal a relevant "field trial" [64]. Reduced fertilization rates $<50 \%$ of mature oocytes in either an IVF- all or an IVF-ICSI Split "trial" indicates a need to apply ICSI for future IVF-related procedures [65]. The idea of applying an IVF-ICSI Split approach as a diagnostic exercise for all IVF-naïve cases of unexplained infertility or intrauterine insemination (IUI) failures has been demonstrated to be a cost-effective approach in the long term [66], one that is favored at PIVET. 


\subsubsection{The IVF-ICSI Split methodology}

Women who are IVF-naïve are encouraged to undertake an IVF-ICSI Split technique on their very first IVF cycle if there is no identifiable male factor and the female infertility is either tubal or unexplained. Such cases could be expected to have normal fertilization potential, albeit that our extensive historical experience exceeding 45 years indicates that poor fertilization (fewer than $50 \%$ of oocytes) and complete failed fertilization will occur in $\sim 15 \%$ (poor fertilization) and $\sim 5 \%$ (failed fertilization) of cases respectively [40]. The IVF-ICSI Split technique is applied when $\geq 4$ mature oocytes are retrieved at OPU and oocytes are distributed randomly for IVF or ICSI, prior to cumulus stripping for the ICSI cases. Where $<4$ mature oocytes are recovered, all oocytes are allocated to ICSI. Although this dataset covers the period from January 2011 to 2019 with outcomes tracked through 2020, the majority of the IVF-ICSI Split group were conducted in the years from 2014 (following unexpected cases of complete fertilization failure) when the practice has become routinely recommended for unexplained infertility.

\subsubsection{Oocyte distributions}

OCCs are graded immediately after OPU applying a longstanding protocol [67] assessing the cumulus cell numbers and tightness of their distribution along with an assessment of the density and tightness of the coronal cells surrounding the oocyte. Thereafter the OCCs are randomly distributed between IVF and ICSI with an equivalent distribution of OCC gradings between the groups. Two embryologists are involved in this process to ensure fairness of distribution between the groups.

\subsubsection{Oocytes allocated to IVF}

Oocytes for IVF were maintained as OCCs and 3-5 hours after OPU were placed in Fertilization Medium (Quinn's Advantage) along with 100,000 spermatozoa prepared 2-4 hours earlier. The sperm preparation involved centrifugation through a 2-layered colloidal silica suspension (Pure sperm 40/80; Nidacon, Sweden). The incubation conditions were within Minc solid-state incubators (COOK, Australia) at $37^{\circ} \mathrm{C}$ in microdroplets under oil (Sage; Origio, Australia) which had been equilibrated overnight and perfused with humidified triple gas - 5\% 02, 6\% CO2 and $89 \%$ N2.

\subsubsection{Oocytes allocated for ICSI}

Three to five hours after OPU, the OCCS for ICSI were prepared by immersion into Hyaluronidase solution (1500 IU Hyalase diluted in Quinn's Advantage Hepes for a final concentration of $80 \mathrm{IU} / \mathrm{ml}$ ) for 30 seconds to disperse the cumulus cells and thereafter subjected to fine needle pipetting to partially strip the remaining cumulus and coronal cells [68]. This was performed within a humidified workstation chamber gassed with 6\% $\mathrm{CO}_{2}$ in air within Quinn's Fertilization medium. Only oocytes at the metaphase II (MII) stage were accepted for ICSI. Those at MI or germinal vesicle (GV) stage, those showing a cracked zona pellucida or those oocytes displaying signs of degeneration are excluded from fertilization attempts.

\subsubsection{Oocyte pronuclear-stage checks}

For both IVF and ICSI fertilized oocytes, the pronuclear-stage checks were performed at $16 \mathrm{~h}$ after overnight incubation in Minc solid-state chambers. Fertilization was reported as 2 PNs per oocytes collected, per oocyte allocated and per injected oocyte (i.e. per metaphase MII oocyte). Oocytes at the germinal vesicle stage or persisting M1 stage were discarded along with those classified as degenerate, denuded or with a fractured zona. All 2 PNs were then placed into cleavage-stage medium (Quinn's Advantage Sequential medium) for culture to Day-3 at which stage ET was considered if there were fewer than 3 high-grade embryos progressing at the 6-8 cell stage. In most cases, there were at least 3 high-grade embryos, and these were transferred to blastocyst culture medium (Quinn's Advantage Sequential medium) for culture through to Day-5 or Day- 6 when ET or cryopreservation by vitrification was performed.

\subsubsection{Oocyte fertilization rates}

For ICSI, the fertilization rate was recorded as those oocytes identified as 2 PNs per oocyte injected. For IVF, the actual fertilization rate was recorded as those oocytes identified as 2 PNS per OCCs inseminated. A second fertilization rate was also recorded being an adjusted rate as those oocytes identified as 2 PNS per OCCs inseminated, applying the same denominator as identified for ICSI. This enables an equivalent fairer comparison for statistical evaluation.

\subsubsection{Intrauterine insemination failures}

Infertile women who had failed to achieve a pregnancy following 2-6 cycles of intrauterine insemination (IUI) were advised to consider ICSI or at least IVF-ICSI Split from our internal studies [45] and indicated by others $[66,68,69]$. From 
internal studies the fertilization rate of cases proceeding to IVF from failed IUI was significantly lower than those directly utilizing ICSI ( $49 \%$ vs $69 \%$; $p<0.001$ ), and occurrences of complete fertilization failure were significantly higher $(13.4 \%$ vs $2.9 \%$; $<0.001)$ causing a change in policy to recommend ICSI or IVF-ICSI Split in such cases.

\subsubsection{Genetic analysis of embryos}

Where preimplantation diagnosis (PGD) and screening (PGS) was applied, the current recommendations are to utilize ICSI to avoid DNA contamination of the embryo biopsy specimens (either blastomeres or trophoblast specimens) from non-fertilizing sperm adherent to the zona pellucida. This is recommended by the ESHRE PGD Consortium [70].

\subsubsection{Cryopreserved gametes}

Both cryopreserved spermatozoa as well as cryopreserved oocytes may show diminished fertilization capacity; in the former because of effects on the acrosomal cap and the latter mainly by effects on the zona pellucida. ICSI was advised when utilizing such cryopreserved gametes, especially when a slow-freeze technique was applied [45].

\subsection{Indications for IVF Only}

Following our earlier study on IVF-ICSI Split, we have reserved the use of IVF Only on cases which exclude male factor infertility, and where satisfactory fertilization has been previously demonstrated in the IVF program or following an IVF-ICSI Split study. Such cases have shown a normal semen profile, as well as a low DNA fragmentation index $(<15 \%)$, along with the collection of $>4$ oocytes from the woman. Nonetheless, occasionally couples with definable male factor infertility have requested IVF Only or IVF-ICSI Split to determine if any IVF ('natural fertilization") embryos could be created. This is enabled at PIVET with first preference for transfer given to the IVF-generated embryos.

\subsection{Freeze-all embryos policy}

To avoid the risk of severe OHSS, the PIVET algorithm is applied for FSH-dosing meaning most women generate $10 \pm 2$ follicles with a matching serum estradiol level (E2) of 10,000 $\pm 2000 \mathrm{pm} / \mathrm{L}$ such that 8-12 oocytes are recovered at oocyte pick-up (OPU). Where $>12$ oocytes are recovered, women commence an increased monitoring protocol and where $\geq 15$ oocytes are recovered, a freeze-all protocol is advised (and is mandatory for $\geq 20$ oocytes) with the luteal phase managed by medroxyprogesterone (MPA) substitution and dopamine receptor antagonist (cabergoline) to minimize the symptoms of OHSS [45].

\subsection{Embryo transfer policy}

Throughout the decade of this study, the protocol has continually advanced towards single blastocyst transfers for both fresh (ET) and cryopreserved (FET) embryos. The criterion is mandatory for all women $<40$ years having up to 3 failed IVF \pm ICSI treatments and remains the preferred protocol for all cases across the treatment ages (to age 50 years). Currently blastocyst transfers are conducted in $>90 \%$ of embryo transfers and the SET rate is $>95 \%$, hence the multiple pregnancy rate is $<5 \%$, mainly monozygotic twinning. The embryo transfers are conducted under trans-vesical abdominal ultrasound using the Cook K-JETS catheter system whereby a mid-high fundal "flash" is identified.

\subsection{Pregnancy definition}

At PIVET pregnancy is denoted by an elevated B-HCG level along with raised estradiol-17B (E2) and progesterone (P4) undertaken $\sim 19$ days after the OPU procedure; denoted as week-4 [45]. At PIVET all pregnancies are monitored each week until week-8 ( 6 weeks post-OPU). Clinical pregnancy is defined by a rising b-HCG from week-4 to week-5, with a definitive ultrasound at week-7 demonstrating an intra-uterine gestational with a viable fetus. Pregnancy losses are defined after week-5 and include pregnancies of unknown location (PUL) which may demonstrate spontaneous hormonal "fade-out" or be given methotrexate (MTX) to enhance the process when a clear intra-uterine gestation cannot be demonstrated. At week-7 pregnancies with a non-viable gestational sac in-utero are encouraged to have an evacuation procedure (along with cytogenetic assessment of the products of conception). Those without an intrauterine gestational sac but persisting BHCG elevation at week are committed to laparoscopic evaluation to manage a probable ectopic gestation (or have intra-cornual MTX administered). PIVET has an active luteal-support approach requiring mid-luteal and early pregnancy P4 levels to record $\geq 60 \mathrm{nmol} / \mathrm{L}$ [45]. High-risk pregnancies and those with threatened miscarriage were offered medroxyprogesterone acetate during the first trimester, continuing to gestational age 35 weeks where pre-term delivery has occurred previously. We have recently published safety and beneficial outcomes from this strategy [71]. 


\subsection{Live birth, pregnancy loss and fetal abnormality}

At PIVET a dedicated midwife manages the pregnancy register and inputs key data into the Filemaker database. She establishes a close relationship with each of the women achieving pregnancy and receives information concerning early pregnancy losses, preterm deliveries, and any adverse event. Within one week after the expected date of delivery (EDD), contact is made with the obstetrician as well as the woman. Live birth (LB) deliveries are categorized as very early preterm, preterm, and full term if the number of gestation days was 140-195, 196-258 and $\geq 259$ days, respectively. Pregnancy loss (PL) is categorized as either early (49 to < 140 days; EPL, miscarriage) or later; sub-categorized as preterm stillbirth, or full-term stillbirth if the fetus was lost/delivered during gestation days 140-258 and $\geq 259$ days, respectively. Each fetal abnormality is categorized according to the International Classification of Disease (ICD) code, and then sub-categorized into the related anatomical system including neural, eye defects, cardiovascular, gastrointestinal, urogenital, musculo-skeletal, skin, chromosomal and any other.

\subsection{Statistical evaluation}

Data from the Filemaker Pro (Apple Inc, USA) database were extracted into Microsoft Excel spreadsheets and assorted according to the relevant tests. The Flow Sheet was created using Microsoft Word for Mac v 16.54. Comparisons between Groups were analyzed in 2x2 contingency tables, mainly by Fisher's exact test, or by Chi-squared applying Yates' continuity correction factor for the larger data sets. Following corrections, probability values of $p<0.05$ were considered significant for any test. Apart from the Flow Sheet, the Figures displayed in this study are derived from Excel v 16.44 (Microsoft 2020), thereafter exported as Word files.

\subsection{Abbreviations applied in this report}

\begin{tabular}{|l|l|}
\hline ART & Assisted reproduction treatments/ technologies \\
\hline Fert Mode & Fertilization by IVF or by ICSI mode \\
\hline Cancel, \% & cancellation rate \\
\hline O & oocyte/s \\
\hline OPU & oocyte pick-up procedure \\
\hline O Utn \% & oocyte utilization rate \\
\hline E Utn \% & embryo utilization rate \\
\hline E Fz \% & embryo cryopreservation (frozen) rate \\
\hline ET & Fresh embryo transfer \\
\hline FET & Frozen embryo transfer \\
\hline Trans. & Transfer/s \\
\hline Preg/In \% & pregnancy rate per initiated cycle \\
\hline Preg/O \% & pregnancy rate per oocyte pick-up (OPU) \\
\hline Preg/ET \% & pregnancy rate per embryo transfer procedure \\
\hline EPL \% & early pregnancy losses from week 5 (miscarriage rate) \\
\hline LB/In \% & live birth rate per initiated cycle \\
\hline LB/O \% & live birth rate per oocyte pick-up (OPU) \\
\hline LB/ET \% & live birth per embryo transfer procedure \\
\hline LBPR & live birth productivity rate (live births per fresh and frozen ETs) \\
\hline IVF & In vitro fertilization (insemination of OCC) \\
\hline ICSI & Intracytoplasmic sperm injection \\
\hline IVF-ICSI Split & OCCs randomised to IVF and ICSI modes \\
\hline OCC & Oocyte cumulus complex \\
\hline MII Oocyte & Oocyte at metaphase II stage; signifies mature \\
\hline 2 PN & Oocyte displaying 2 pronuclei; signifies fertilized \\
\hline AFC & antral follicle count (small follicles <10mm) \\
\hline AMH & anti-Mullerian hormone \\
\hline BMI & Body mass index kg/m ${ }^{2}$ \\
\hline
\end{tabular}




\begin{tabular}{|l|l|}
\hline FSH & Follicle stimulating hormone (serum measurement) \\
\hline PIVET & registered acronym from programmed IVF \& ET \\
\hline Stats & statistics \\
\hline n.s. & not significant \\
\hline yrs & years \\
\hline$\#$ & total \\
\hline
\end{tabular}

\section{Results}

Across the period 2011 to 2019, 2376 infertile women initiated 3767 IVF \pm ICSI treatment cycles at PIVET where autologous oocytes were utilized to generate embryos intended for transfer with the view of generating healthy children for oneself. (Whilst this includes women accessing donor sperm, the 242 treatment cycles from 233 women utilizing the IVF-ICSI Split protocol is reserved for those women with a male partner who has a normal semen analysis and the couple being categorized as having unexplained infertility). This included the idea of cryopreservation of embryos and maximizing the productivity rate from a single initiated IVF cycle as reported in this study. Therefore, cycles involving donor sperm, donor oocytes and donor embryos have been excluded. Also cycles involving oocyte or embryo banking for future potential use have also been excluded.

Figure 1 Flowsheet depicting the derivation of 3637 IVF \pm ICSI treatment cycles from 2376 women across the period 1 January 2011 until 31 December 2019 with pregnancy outcomes tracked through 2020. The treatments are subcategorized according to the insemination process; IVF, ICSI or IVF-ICSI Split modes. The IVF-ICSI Split cycles are further categorized according to those reaching a fresh ET procedure and those having FET procedures following a freeze-all cycle.

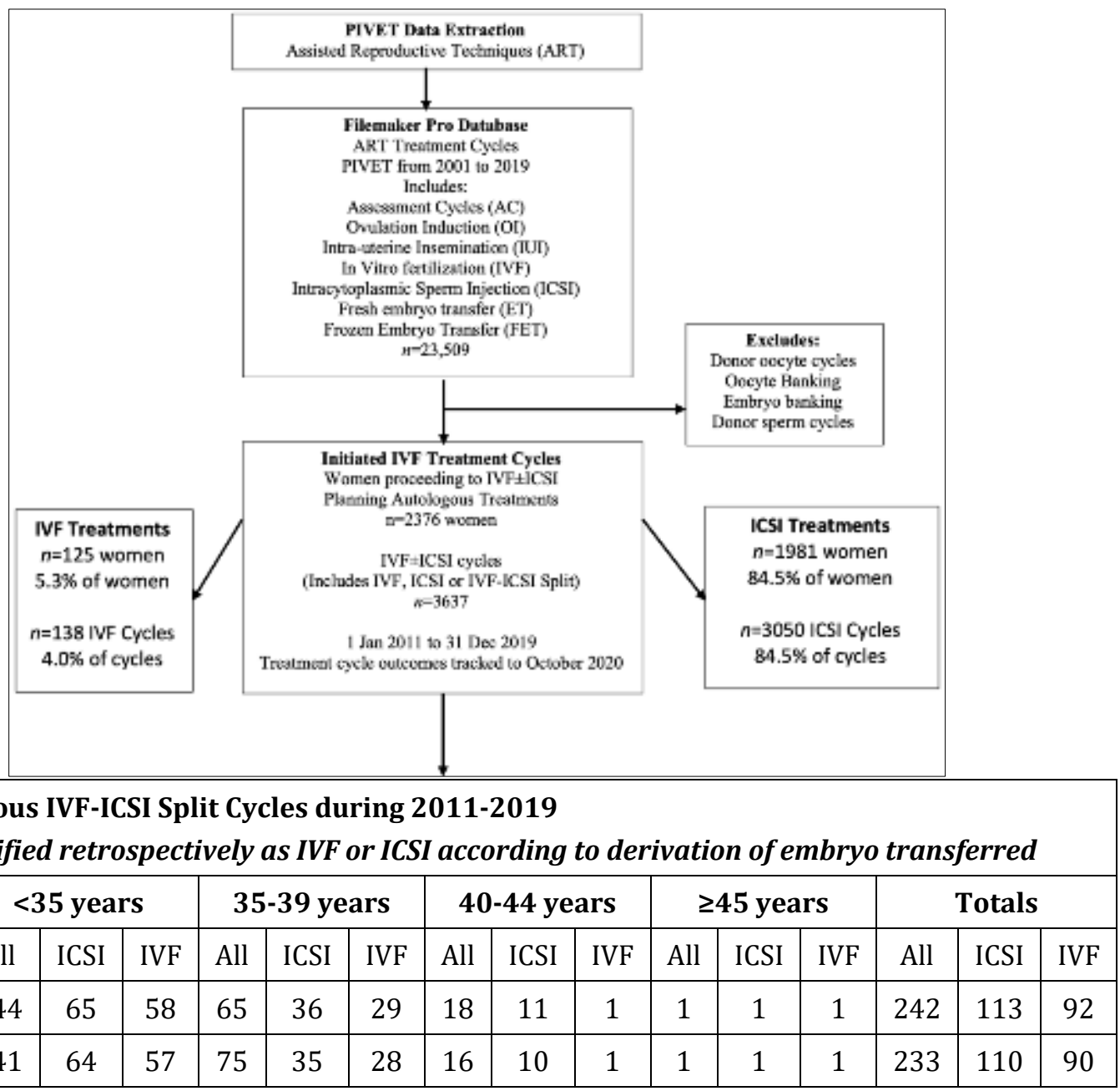




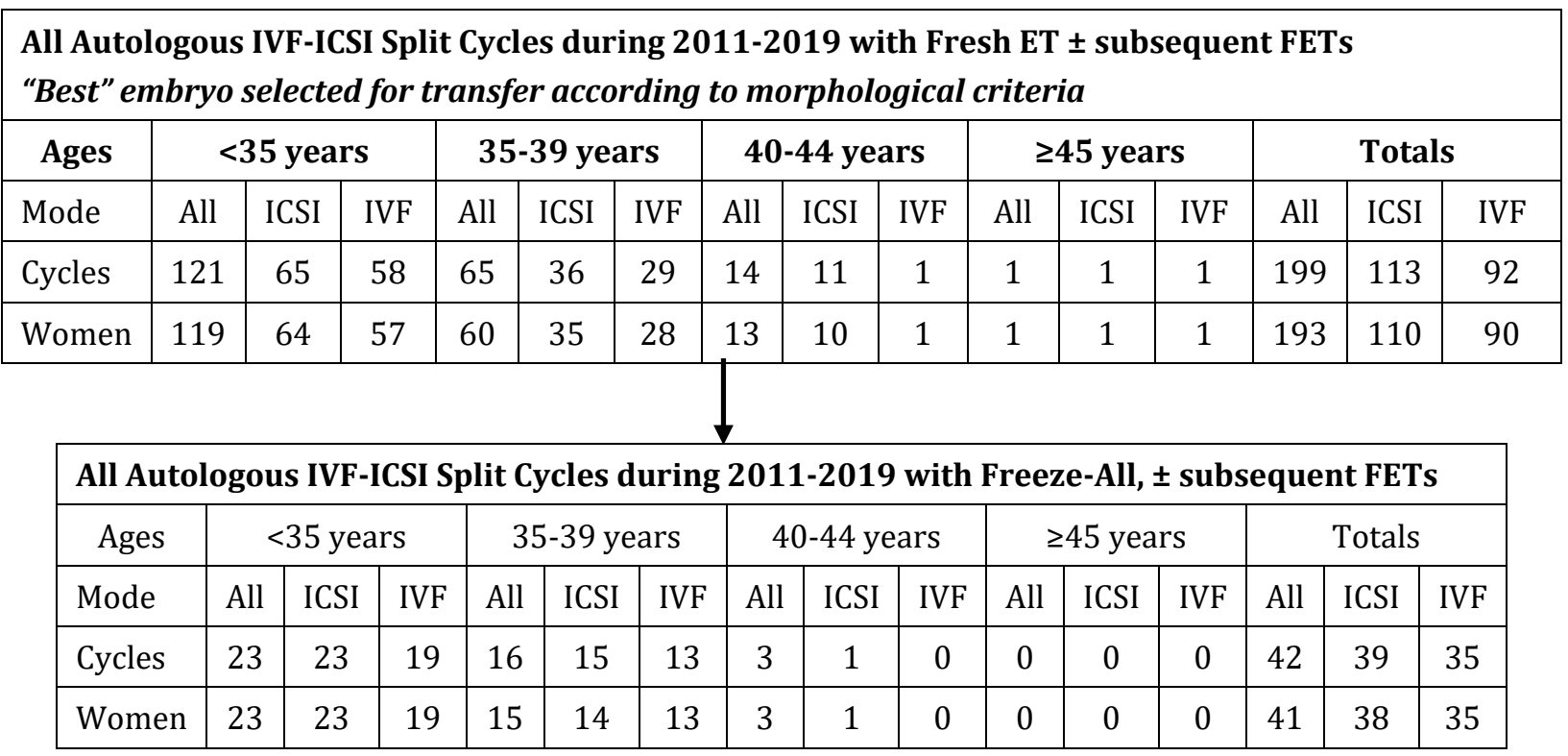

From the Flow sheet (Figure1) most treatments were undertaken utilizing the ICSI technique for fertilization - 1981 of the women (84.5\%) initiated 3050 cycles (88.9\%) for insemination by ICSI. Hence, only a smaller proportion of women conducted their treatments by IVF insemination only - 125 of the women (5.3\%) initiated 138 cycles (4.0\%) for insemination by traditional IVF. These women had mostly been shown to have satisfactory fertilization $(\geq 50 \%$ of oocytes) on previous treatment cycles. Therefore, IVF-naïve women with tubal or unexplained infertility were encouraged to undertake IVF-ICSI Split treatments hence 233 women (9.8\%) initiated 242 (6.7\%) treatment cycles where $\geq 4$ oocytes were randomly allocated to either IVF or ICSI insemination. Cases are then categorized according to the derivation of the embryo transferred (being IVF-generated or ICSI-generated). The number of women and their IVF \pm ICSI cycles are shown in specific display boxes (covering the 242 total treatment cycles, the 199 treatment cycles having a fresh embryo transfer, and the 42 treatment cycles having freeze-all embryos, some of which proceeded to subsequent FETs).

Table 2 Summarizes the raw data outcomes of 242 IVF-ICSI Split Cycles from 233 women who reached OPU

\begin{tabular}{|l|c|c|c|c|c|}
\hline \multicolumn{7}{|c|}{ Autologous IVF-ICSI Split Cycles during 2011-2019 - numbers } \\
\hline Age groups & $<\mathbf{3 5}$ yrs & $\mathbf{3 5 - 3 9}$ yrs & $\mathbf{4 0 - 4 4}$ yrs & $\mathbf{2 4 5}$ yrs & Total \\
\hline Treatment Cycles & 144 & 79 & 18 & 1 & 242 \\
\hline Women treated & 141 & 75 & 16 & 1 & 233 \\
\hline Cancelled cycles & 0 & 0 & 0 & 0 & 0 \\
\hline \#OPU & 144 & 79 & 18 & 1 & 242 \\
\hline Oocytes Collected (OCCs) & 2046 & 1073 & 223 & 4 & 3346 \\
\hline Oocytes Inseminated IVF & 965 & 496 & 107 & 2 & 1570 \\
\hline Oocytes Injected ICSI & 873 & 446 & 88 & 2 & 1409 \\
\hline \#2PN & 1249 & 631 & 124 & 2 & 2019 \\
\hline 2PN (ICSI Only) & 729 & 360 & 64 & 1 & 1154 \\
\hline 2PN (IVF Only) & 521 & 283 & 60 & 1 & 865 \\
\hline \#Freeze-all embryos & 23 & 16 & 3 & 0 & 42 \\
\hline \#ET cycles (Fresh ETs) & 121 & 64 & 14 & 1 & 200 \\
\hline
\end{tabular}




\begin{tabular}{|c|c|c|c|c|c|}
\hline Total Fresh embryos transf. & 124 & 69 & 17 & 2 & 212 \\
\hline - $\quad$ Fresh ICSI transfer & 65 & 40 & 12 & 1 & 118 \\
\hline - $\quad$ Fresh IVF transfer. & 59 & 29 & 5 & 1 & 94 \\
\hline \# Embryos Frozen Total & 519 & 251 & 27 & 0 & 797 \\
\hline - $\quad$ ICSI Embryos Frozen & 312 & 147 & 11 & 0 & 470 \\
\hline - $\quad$ IVF Embryos Frozen & 207 & 104 & 16 & 0 & 327 \\
\hline FET Cycles & 167 & 88 & 13 & 0 & 268 \\
\hline Total embryos at FETs & 168 & 90 & 15 & 0 & 273 \\
\hline - $\quad$ ICSI embryo FETs & 108 & 54 & 6 & 0 & 168 \\
\hline - $\quad$ IVF embryo FETs & 168 & 100 & 16 & 0 & 284 \\
\hline Total Transfers Fresh/Frozen & 288 & 151 & 27 & 2 & 468 \\
\hline Total Embryos F/Fz transfers & 291 & 158 & 32 & 2 & 483 \\
\hline \#Pregs Fresh only & 61 & 19 & 3 & 0 & 83 \\
\hline - $\quad$ Pregs Fresh (ICSI) & 30 & 11 & 1 & 0 & 42 \\
\hline - $\quad$ Pregs Fresh (IVF) & 31 & 8 & 2 & 0 & 41 \\
\hline \#Pregs Frozen only & 84 & 41 & 2 & 0 & 127 \\
\hline - $\quad$ Pregs Frozen (ICSI) & 66 & 36 & 1 & 0 & 103 \\
\hline - $\quad$ Pregs Frozen (IVF) & 18 & 5 & 1 & 0 & 24 \\
\hline \#Pregs Fresh/frozen & 145 & 60 & 5 & 0 & 210 \\
\hline \#Miscarriages & 31 & 14 & 2 & 0 & 47 \\
\hline \#Live Births from fresh ETs & 54 & 15 & 1 & 0 & 70 \\
\hline \#Babies from Fresh ETs & 55 & 16 & 1 & 0 & 72 \\
\hline - $\quad$ Live Births (ICSI) & 28 & 8 & 0 & 0 & 36 \\
\hline - $\quad$ Live Births (IVF) & 26 & 7 & 1 & 0 & 34 \\
\hline \#Live Births from FETs & 61 & 31 & 2 & 0 & 94 \\
\hline \#Babies from FETs & 66 & 31 & 2 & 0 & 97 \\
\hline - $\quad$ Livebirths (ICSI) Fz & 36 & 21 & 1 & 0 & 58 \\
\hline - $\quad$ Livebirths (IVF) Fz & 30 & 19 & 1 & 0 & 50 \\
\hline \#LiveBirths Fresh/Fz 1 1 st Trans. & 115 & 46 & 3 & 0 & 164 \\
\hline Total babies Fresh/Fz $1^{\text {st }}$ Trans. & 121 & 46 & 3 & 0 & 170 \\
\hline
\end{tabular}

The laboratory data including oocyte numbers retrieved at OPU and the insemination outcomes (IVF vs ICSI) are depicted in Table 2. It can be seen that 3346 OCCs were recovered (13.8 oocytes/ OPU) and 2979 were deemed suitable for a fertilization procedure, allocating 1570 to IVF and 1409 to ICSI. It is shown that the 2PN (pronuclear stage) which identified normal fertilization was identified in 2019 of the 2979 oocytes inseminated (by IVF or ICSI) being 67.8\% overall. This means $11.0 \%$ oocytes are not inseminated as they have not reached the MII stage of maturity or have fractured zonae, possibly a reflection of using the double-lumen needle for follicle flushing which enables a very high oocyte recovery rate approaching $100 \%$ for follicles $\geq 14 \mathrm{~mm}$ [7]. However, the fertilization rate varied from $8652 \mathrm{PNs}$ arising from 1570 oocytes undergoing IVF insemination (55.1\%) to 1154 of 1409 oocytes (81.9\%) having ICSI insemination. For comparable matching of oocytes, the IVF rates are adjusted to 865 of 1409 oocytes (61.4\%) to match those MII oocytes defined for ICSI. Irrespective of the adjustment, these fertilization rates were highly significant across 
the entire age range groupings, in favor of ICSI $(\mathrm{p}<0.0001)$. However, when analyzing the fertilization rates for individual age groupings, this highly significant difference was shown only for those women aged <40 years (Table 3 ).

Table 3 Shows the rates and statistical significance of the outcomes of 242 IVF-ICSI Split Cycles from 233 women who reached OPU

\begin{tabular}{|c|c|c|c|c|c|c|c|c|c|c|c|c|c|c|c|}
\hline \multicolumn{16}{|c|}{ All Autologous IVF-ICSI Split Cycles during 2011-2019 - rates \% } \\
\hline Ages & \multicolumn{3}{|c|}{$<35$ years } & \multicolumn{3}{|c|}{$35-39$ years } & \multicolumn{3}{|c|}{$40-44$ years } & \multicolumn{3}{|c|}{$\geq 45$ years } & \multicolumn{3}{|c|}{ Totals } \\
\hline Fert mode & All & ICSI & IVF & All & ICSI & IVF & All & ICSI & IVF & All & ICSI & IVF & All & ICSI & IVF \\
\hline Cycles & 144 & 65 & 58 & 65 & 36 & 29 & 18 & 11 & 1 & 1 & 1 & 1 & 242 & 113 & 92 \\
\hline Women & 141 & 64 & 57 & 75 & 35 & 28 & 16 & 10 & 1 & 1 & 1 & 1 & 233 & 110 & 90 \\
\hline Cancel \% & 0.0 & 0.0 & 0.0 & 0.0 & 0.0 & 0.0 & 0.0 & 0.0 & 0.0 & 0.0 & 0.0 & 0.0 & 0.0 & 0.0 & 0.0 \\
\hline O/OPU & 14.2 & 12.1 & 12.9 & 13.6 & 11.6 & 12.2 & 12.4 & 11.5 & 15.5 & 4.0 & 4.0 & 4.0 & 13.8 & 11.8 & 12.7 \\
\hline Fert \%* & 61.0 & 84.1 & 64.6 & 58.8 & 83.8 & 66.7 & 55.6 & 76.4 & 60.0 & 50.0 & 50.0 & 50.0 & 60.0 & 83.2 & 65.4 \\
\hline Stats: & \multicolumn{3}{|c|}{$p<0.0001$} & \multicolumn{3}{|c|}{$p<0.0001$} & \multicolumn{3}{|c|}{ n.s. } & \multicolumn{3}{|c|}{ n.s. } & \multicolumn{3}{|c|}{$p<0.0001$} \\
\hline O Utn \% & 31.3 & 31.3 & 34.9 & 29.6 & 26.7 & 30.8 & 19.7 & 20.5 & 22.6 & 50.0 & 50.0 & 50.0 & 30.0 & 46.8 & 42.7 \\
\hline E Utn \% & 51.3 & 51.9 & 53.4 & 50.4 & 46.9 & 49.8 & 35.5 & 34.7 & 42.4 & 100 & 100 & 100 & 50.1 & 48.9 & 51.9 \\
\hline $\mathrm{E} \mathrm{Fz} \%$ & 41.5 & 37.9 & 41.1 & 39.6 & 29.7 & 36.1 & 21.8 & 17.3 & 24.2 & 0.0 & 0.0 & 0.0 & 39.6 & 33.4 & 38.8 \\
\hline PP/ ET \% & 49.8 & 42.6 & 56.8 & 38.0 & 44.9 & 33.8 & 15.6 & 10.0 & 25.0 & 0.0 & 0.0 & 0.0 & 43.5 & 40.1 & 46.8 \\
\hline PP/Init \% & 100.7 & 89.2 & 108.6 & 75.9 & 86.1 & 75.9 & 27.8 & 18.2 & 50.0 & 0.0 & 0.0 & 0.0 & 86.8 & 80.5 & 94.6 \\
\hline PP/OPU \% & 100.7 & 89.2 & 108.6 & 86.1 & 86.1 & 75.9 & 27.8 & 18.2 & 50.0 & 0.0 & 0.0 & 0.0 & 86.8 & 80.5 & 94.6 \\
\hline EPL \% & 21.4 & 12.1 & 25.4 & 23.3 & 27.3 & 27.3 & 40.0 & 50.0 & 50.0 & 0.0 & 0.0 & 0.0 & 22.4 & 18.7 & 26.4 \\
\hline Stats: & \multicolumn{3}{|c|}{ n.s. } & \multicolumn{3}{|c|}{ n.s. } & \multicolumn{3}{|c|}{ n.s. } & \multicolumn{3}{|c|}{ n.s. } & \multicolumn{3}{|c|}{ n.s. } \\
\hline LBR/ET\% & 39.2 & 37.5 & 42.3 & 29.1 & 31.9 & 24.6 & 9.4 & 5.0 & 12.5 & 0.0 & 0.0 & 0.0 & 33.7 & 32.6 & 34.4 \\
\hline Stats: & \multicolumn{3}{|c|}{ n.s. } & \multicolumn{3}{|c|}{ n.s. } & \multicolumn{3}{|c|}{ n.s. } & \multicolumn{3}{|c|}{ n.s. } & \multicolumn{3}{|c|}{ n.s. } \\
\hline LBP/ In \% & 79.2 & 78.5 & 81.0 & 58.2 & 55.2 & 55.2 & 16.7 & 9.1 & 25.0 & 0.0 & 0.0 & 0.0 & 67.4 & 65.5 & 69.6 \\
\hline LBP/OPU & 79.2 & 78.5 & 81.0 & 58.2 & 55.2 & 55.2 & 16.7 & 9.1 & 25.0 & 0.0 & 0.0 & 0.0 & 67.4 & 65.5 & 69.6 \\
\hline Stats: & \multicolumn{3}{|c|}{ n.s. } & \multicolumn{3}{|c|}{ n.s. } & \multicolumn{3}{|c|}{ n.s. } & \multicolumn{3}{|c|}{ n.s. } & \multicolumn{3}{|c|}{ n.s. } \\
\hline
\end{tabular}

*Fertilization rate for IVF: denominator adjusted from total 425 oocytes, to 358 oocytes reflecting likely number of MII oocytes (presumed to be the same as ICSI group).

The utilization rates of those embryos arising from the fertilized oocytes are shown in Table 3 which denotes them as either IVF or ICSI-derived. A proportion of the embryos were transferred in fresh cycles (total $\mathrm{n}=200$ embryos in 200 ET cycles) whilst a larger proportion were cryopreserved (total $n=797$ embryos; 470 derived from ICSI and 327 derived from IVF). From the combined total of 468 ET and FET transfers a total 483 embryos were transferred indicating a $96.9 \%$ SET rate. The $3.1 \%$ of women having 2 embryos transferred were all aged over 40 years with limited opportunity for repeat ART procedures. The embryo utilization rates across the age groups are shown in Table 3 where the rates were $\sim 45 \%$ with no significant differences across the groups $<45$ years. However, in the oldest group of women $\geq 45$ years there was an apparently higher utilization for IVF-derived embryos, but this is likely an aberration related to the low numbers in this group as well as the determined attempt to give such women "every chance" despite low quality of their embryos. With respect to pregnancies arising from the respective ICSI-generated or IVF-generated embryos, there were no significant differences. This finding carried through to pregnancy outcomes with similar rates of miscarriage and live births across the age groupings as well as the total population of 233 women having 242 treatment cycles. 
Table 4 Summarizes the raw data outcomes of 200 IVF-ICSI Split Cycles from 193 women who reached the stage of a fresh ET. The data excludes 42 women who had cycles where all embryos were cryopreserved (freeze-all)

\begin{tabular}{|c|c|c|c|c|c|}
\hline \multicolumn{6}{|c|}{ Autologous IVF-ICSI Split Cycles excluding 42 Freeze-alls - numbers } \\
\hline Age groups & $<35$ yrs & $35-39$ yrs & 40-44 yrs & $\geq 45$ yrs & Total \\
\hline Treatment Cycles & 121 & 63 & 14 & 2 & 200 \\
\hline Women treated & 119 & 60 & 13 & 1 & 193 \\
\hline Cancelled cycles & 0 & 0 & 0 & 1 & 1 \\
\hline \#OPU & 121 & 63 & 14 & 1 & 199 \\
\hline Oocytes Collected (OCCs) & 1503 & 752 & 182 & 4 & 2441 \\
\hline Oocytes Inseminated IVF & 710 & 346 & 86 & 2 & 1144 \\
\hline Oocytes Injected ICSI & 657 & 318 & 73 & 2 & 1050 \\
\hline \#2PN & 940 & 444 & 105 & 2 & 1504 \\
\hline - $\quad$ \#2PN (ICSI Only) & 546 & 258 & 53 & 1 & 858 \\
\hline - $\quad$ \#2PN (IVF Only) & 395 & 198 & 52 & 1 & 646 \\
\hline \#Freeze-all embryos & 0 & 0 & 0 & 0 & 0 \\
\hline \#ET cycles (Fresh ETs) & 121 & 63 & 14 & 1 & 199 \\
\hline Total Fresh embryos transf. & 125 & 68 & 17 & 2 & 212 \\
\hline - Fresh ICSI transfer. & 65 & 40 & 12 & 1 & 118 \\
\hline - $\quad$ Fresh IVF transfer. & 59 & 29 & 5 & 1 & 94 \\
\hline \# Embryos Frozen Total & 370 & 150 & 21 & 0 & 541 \\
\hline ICSI Embryos Frozen & 226 & 89 & 8 & 0 & 323 \\
\hline IVF Embryos Frozen & 144 & 61 & 13 & 0 & 218 \\
\hline FET Cycles & 118 & 62 & 8 & 0 & 188 \\
\hline Total Frozen embryos trans. & 119 & 62 & 9 & 0 & 190 \\
\hline - $\quad$ Frozen ICSI embryos trans. & 84 & 39 & 3 & 0 & 126 \\
\hline - $\quad$ Frozen IVF embryos trans. & 108 & 57 & 13 & 0 & 178 \\
\hline Total Transfers Fresh/frozen & 242 & 130 & 26 & 2 & 400 \\
\hline Total Embryos F/fr transfer. & 492 & 217 & 38 & 2 & 749 \\
\hline \#Pregs Fresh only & 61 & 19 & 3 & 0 & 83 \\
\hline - $\quad$ Pregs Fresh (ICSI) & 30 & 11 & 1 & 0 & 42 \\
\hline - $\quad$ Pregs Fresh (IVF) & 31 & 8 & 2 & 0 & 41 \\
\hline \#Pregs Frozen only & 57 & 33 & 1 & 0 & 91 \\
\hline - $\quad$ Pregs Frozen (ICSI) & 46 & 29 & 0 & 0 & 75 \\
\hline - $\quad$ Pregs Frozen (IVF) & 11 & 4 & 1 & 0 & 16 \\
\hline \#Pregs Fresh/frozen & 118 & 52 & 4 & 0 & 174 \\
\hline \#Miscarriages & 23 & 13 & 2 & 0 & 38 \\
\hline \#Live Births from fresh ETs & 54 & 15 & 1 & 0 & 70 \\
\hline \#Babies from fresh ETs & 55 & 15 & 1 & 0 & 71 \\
\hline
\end{tabular}




\begin{tabular}{|c|c|c|c|c|c|}
\hline - Live Births (ICSI) Fresh & 28 & 8 & 0 & 0 & 36 \\
\hline - Live Births (IVF) Fresh & 26 & 7 & 1 & 0 & 34 \\
\hline \#Live Births from FETs & 42 & 24 & 1 & 0 & 67 \\
\hline Total Babies from FETs & 43 & 25 & 1 & 0 & 69 \\
\hline - Live Births (ICSI) Fz & 19 & 15 & 0 & 0 & 34 \\
\hline - Live Births (IVF) Fz & 16 & 16 & 1 & 0 & 33 \\
\hline \#LiveBirths from Fresh/Fz & 96 & 39 & 2 & 0 & 137 \\
\hline - Live Births (ICSI) Fr/Fz & 47 & 23 & 0 & 0 & 70 \\
\hline - Live Births (IVF) Fr/Fz & 42 & 23 & 2 & 0 & 67 \\
\hline Total babies from Fresh/Fz & 91 & 47 & 2 & 0 & 140 \\
\hline
\end{tabular}

Analyzing the laboratory and clinical outcomes from those women who had fresh embryo transfers separately (i.e., excluding those who had a high response with oocyte numbers $>15$, resulting in a "freeze-all" cycle), in Table 4 it can be seen that 193 women reached the stage of OPU but one cycle in a woman aged 45-years, was cancelled at the stage of Trigger because of an uncertain response with probable premature ovulation. The 199 cycles yielded 2441 OCCs of which 1050 were allocated to ICSI and 1144 were inseminated by IVF (10.1\% discarded. Applying the same denominator of 1050, the fertilization rates were 858; 81.7\% for ICSI and 646; $61.5 \%$ for IVF ( $\mathrm{p}<0.0001$ ).

Table 5 Shows the rates and statistical significance of the outcomes of 200 IVF-ICSI Split Cycles from 193 women who reached the stage of a fresh ET. The data excludes 42 women who had cycles where all embryos were cryopreserved (freeze-all)

\begin{tabular}{|c|c|c|c|c|c|c|c|c|c|c|c|c|c|c|c|}
\hline \multicolumn{16}{|c|}{ Autologous IVF-ICSI Split Cycles excluding 42 Freeze-all - Rates \% } \\
\hline Ages & \multicolumn{3}{|c|}{$<35$ years } & \multicolumn{3}{|c|}{$35-39$ years } & \multicolumn{3}{|c|}{ 40-44 years } & \multicolumn{3}{|c|}{$\geq 45$ years } & \multicolumn{3}{|c|}{ Totals } \\
\hline Adj & All & ICSI & IVF & All & ICSI & IVF & All & ICSI & IVF & All & ICSI & IVF & All & ICSI & IVF \\
\hline Cycles & 121 & 65 & 58 & 65 & 36 & 29 & 14 & 11 & 1 & 2 & 1 & 1 & 200 & 113 & 92 \\
\hline Women & 119 & 64 & 57 & 60 & 35 & 28 & 13 & 10 & 1 & 2 & 1 & 1 & 193 & 110 & 90 \\
\hline Canc \% & 0.0 & 0.0 & 0.0 & 0.0 & 0.0 & 0.0 & 0.0 & 0.0 & 0.0 & 0.5 & 0.0 & 0.0 & 0.5 & 0.0 & 0.0 \\
\hline 0/OPU & 12.4 & 12.1 & 12.9 & 11.9 & 11.6 & 12.2 & 13.0 & 11.5 & 15.5 & 4.0 & 4.0 & 4.0 & 12.3 & 11.8 & 12.7 \\
\hline FertRate $\%$ & 62.5 & 84.1 & 64.6 & 59.0 & 83.8 & 66.7 & 57.7 & 76.4 & 69.0 & 50.0 & 50.0 & 50.0 & 61.1 & 83.2 & 65.4 \\
\hline Stats: & \multicolumn{3}{|c|}{$p<0.0001$} & \multicolumn{3}{|c|}{$p<0.0001$} & \multicolumn{3}{|c|}{ n.s. } & \multicolumn{3}{|c|}{ n.s. } & \multicolumn{3}{|c|}{$p<0.0001$} \\
\hline O Utn \% & 32.7 & 31.3 & 34.9 & 28.9 & 26.7 & 30.8 & 20.9 & 20.5 & 22.6 & 50.0 & 50.0 & 50.0 & 30.7 & 28.9 & 33.1 \\
\hline E Utn \% & 52.3 & 51.9 & 53.4 & 48.9 & 46.9 & 49.8 & 36.2 & 34.7 & 42.4 & 100 & 100 & 100 & 50.2 & 48.9 & 51.9 \\
\hline E Fz \% & 39.3 & 37.9 & 41.1 & 33.6 & 29.7 & 36.1 & 20.0 & 17.3 & 24.2 & 0.0 & 0.0 & 0.0 & 36.2 & 33.4 & 38.8 \\
\hline PP/ET \% & 48.8 & 42.6 & 56.8 & 40.0 & 44.9 & 33.8 & 15.4 & 10.0 & 25.0 & 0.0 & 0.0 & 0.0 & 43.5 & 40.1 & 46.8 \\
\hline PP/Init \% & 97.5 & 89.2 & 108.6 & 82.5 & 86.1 & 75.9 & 28.6 & 18.2 & 50.0 & 0.0 & 0.0 & 0.0 & 87.4 & 80.5 & 94.6 \\
\hline PP/ OPU \% & 97.5 & 89.2 & 108.6 & 82.5 & 86.1 & 75.9 & 28.6 & 18.2 & 50.0 & 0.0 & 0.0 & 0.0 & 87.4 & 80.5 & 94.6 \\
\hline EPL \% & 19.5 & 12.1 & 25.4 & 25.0 & 27.3 & 27.3 & 50.0 & 50.0 & 50.0 & 0.0 & 0.0 & 0.0 & 21.8 & 17.6 & 26.4 \\
\hline Stats: & \multicolumn{3}{|c|}{ n.s. } & \multicolumn{3}{|c|}{ n.s. } & \multicolumn{3}{|c|}{ n.s. } & \multicolumn{3}{|c|}{ n.s. } & \multicolumn{3}{|c|}{ n.s. } \\
\hline LBP/ET \% & 39.3 & 37.5 & 42.3 & 30.0 & 33.3 & 24.6 & 7.7 & 5.0 & 12.5 & 0.0 & 0.0 & 0.0 & 34.0 & 33.0 & 34.4 \\
\hline Stats: & \multicolumn{3}{|c|}{ n.s. } & \multicolumn{3}{|c|}{ n.s. } & \multicolumn{3}{|c|}{ n.s. } & \multicolumn{3}{|c|}{ n.s. } & \multicolumn{3}{|c|}{ n.s. } \\
\hline
\end{tabular}




\begin{tabular}{|l|l|l|l|l|l|l|l|l|l|l|l|l|l|l|l|}
\hline LBP/Init \% & 79.2 & 78.5 & 81.0 & 58.2 & 55.2 & 55.2 & 16.7 & 9.1 & 25.0 & 0.0 & 0.0 & 0.0 & 67.4 & 65.5 & 69.6 \\
\hline $\begin{array}{l}\text { LBP/OPU } \\
\%\end{array}$ & 78.5 & 78.5 & 81.0 & 61.9 & 63.9 & 55.2 & 14.3 & 9.1 & 25.0 & 0.0 & 0.0 & 0.0 & 68.3 & 66.4 & 69.6 \\
\hline Stats: & \multicolumn{3}{|c|}{ n.s. } & \multicolumn{3}{|c|}{ n.s. } & \multicolumn{3}{c|}{ n.s. } & \multicolumn{3}{|c|}{ n.s. } & \multicolumn{3}{c|}{ n.s. } \\
\hline
\end{tabular}

Despite the significantly higher fertilization numbers and rates shown in Table 4, the embryo utilization rates (embryos resulting in ETs or cryopreserved) were similar between the IVF-generated and the ICSI-generated. Hence, as shown in Table 5, the pregnancy productivity rates (from fresh ETs and FETs), the miscarriage rates (EPLs) and the live birth productivity rates were not significantly different, neither for the total group nor the individual age groupings.

Table 6 Summarizes the raw data outcomes of 42 IVF-ICSI Split Cycles from 41 women who reached OPU but where all resulting embryos were cryopreserved (freeze-all) because of a high risk of OHSS. Such women subsequently had FET procedures

\begin{tabular}{|c|c|c|c|c|c|}
\hline \multicolumn{6}{|c|}{ Autologous IVF-ICSI Split Cycles: 42 Freeze-all - numbers } \\
\hline Age groups & $<35$ yrs & $35-39$ yrs & $40-44$ yrs & $\geq 45$ yrs & Total \\
\hline Treatment Cycles & 23 & 16 & 3 & 0 & 42 \\
\hline Women treated & 23 & 15 & 3 & 0 & 41 \\
\hline Cancelled cycles & 0 & 0 & 0 & 0 & 0 \\
\hline \#OPU & 23 & 16 & 3 & 0 & 42 \\
\hline Oocytes Collected (OCCs) & 543 & 321 & 39 & 0 & 903 \\
\hline Oocytes/ OPU & 23.6 & 20.1 & 13.0 & 0 & 21.5 \\
\hline Oocytes Injected by ICSI & 216 & 128 & 14 & 0 & 358 \\
\hline Oocytes Inseminated by IVF & 255 & 150 & 20 & 0 & 425 \\
\hline \#2PN & 309 & 187 & 19 & 0 & 515 \\
\hline$-\quad$ \#2PN (ICSI) & 183 & 102 & 11 & 0 & 296 \\
\hline - $\quad$ \#2PN (IVF Only) * & 126 & 85 & 8 & 0 & 219 \\
\hline Fertilization rate $\%$ & 65.6 & 67.2 & 55.9 & 0 & 65.7 \\
\hline - $\quad$ Fert by ICSI $(296 / 358)$ & 84.7 & 79.7 & 78.6 & 0 & 82.7 \\
\hline - $\quad$ Fert by IVF $(219 / 358)^{*}$ & 58.3 & 66.4 & 57.1 & 0 & 61.2 \\
\hline \# Embryos Frozen & 149 & 101 & 6 & 0 & 256 \\
\hline - $\quad$ ICSI Embryos Fz & 86 & 58 & 3 & 0 & 147 \\
\hline - $\quad$ IVF Embryos Fz & 63 & 43 & 3 & 0 & 109 \\
\hline FET Cycles & 49 & 26 & 5 & 0 & 80 \\
\hline Total Frozen Embryos Transfers & 49 & 28 & 6 & 0 & 83 \\
\hline - $\quad$ FET ICSI embryos & 24 & 15 & 3 & 0 & 42 \\
\hline - $\quad$ FET IVF embryos & 60 & 43 & 3 & 0 & 106 \\
\hline \#Pregs Frozen only & 27 & 8 & 1 & 0 & 36 \\
\hline - $\quad$ Pregs Frozen (ICSI) & 20 & 7 & 1 & 0 & 28 \\
\hline - $\quad$ Pregs Frozen (IVF) & 7 & 1 & 0 & 0 & 8 \\
\hline \#Miscarriages & 8 & 1 & 0 & 0 & 9 \\
\hline
\end{tabular}




\begin{tabular}{|c|r|r|r|r|r|}
\hline \#Live Births from FETs & 19 & 7 & 1 & 0 & 27 \\
\hline - Livebirths (ICSI) Frozen & 9 & 4 & 1 & 0 & 14 \\
\hline - Livebirths (IVF) Frozen & 10 & 3 & 0 & 0 & 13 \\
\hline Total Babies from FETs & 21 & 7 & 1 & 0 & 29 \\
\hline
\end{tabular}

*Fertilization rate for IVF: denominator adjusted from total 425 oocytes, to 358 oocytes reflecting likely number of MII oocytes (presumed to be the same as ICSI group).

Separately analyzing the data from those women who had a "freeze-all" treatment cycle, Table 6 indicates that 41 women had 42 cycles where 903 OCCs were recovered at OPU ( 21.5 oocytes/ OPU). These cycles were deemed high risk for OHSS. Of the 903 OCCs, 783 oocytes were utilized with 358 allocated to ICSI and 425 allocated to insemination by IVF $13.3 \%$ oocytes discarded. Applying the same ICSI denominator of 358, the fertilization rates were $82.7 \%$ for ICSI and $61.2 \%$ for IVF $(\mathrm{p}<0.0001)$.

Table 7 Shows the rates and statistical significance of the outcomes of 42 IVF-ICSI Split Cycles from 41 women who reached OPU but where all resulting embryos were cryopreserved (freeze-all) because of a high risk of OHSS. Such women subsequently had FET procedures

\begin{tabular}{|c|c|c|c|c|c|c|c|c|c|c|c|c|c|c|c|}
\hline \multicolumn{16}{|c|}{ Autologous IVF-ICSI Split Cycles: 42 Freeze-all - Rates } \\
\hline Ages & \multicolumn{3}{|c|}{$<35$ years } & \multicolumn{3}{|c|}{$35-39$ years } & \multicolumn{3}{|c|}{ 40-44 years } & \multicolumn{3}{|c|}{$\geq 45$ years } & \multicolumn{3}{|c|}{ Totals } \\
\hline Fert Mode & All & ICSI & IVF & All & ICSI & IVF & All & ICSI & IVF & All & ICSI & IVF & All & ICSI & IVF \\
\hline Cycles & 23 & 23 & 19 & 16 & 15 & 13 & 3 & 1 & 0 & 0 & 0 & 0 & 42 & 39 & 35 \\
\hline Women & 23 & 23 & 19 & 15 & 14 & 13 & 3 & 1 & 0 & 0 & 0 & 0 & 41 & 38 & 35 \\
\hline Cancel \% & 0 & 0 & 0 & 0 & 0 & 0 & 0 & 0 & 0 & 0 & 0 & 0 & 0 & 0 & 0 \\
\hline 0/OPU \% & 23.6 & 23.6 & 24.4 & 20.1 & 21.1 & 21.9 & 13.0 & 23.0 & 13.0 & 0 & 0 & 0 & 21.5 & 22.6 & 22.5 \\
\hline Fert Rate \% & 65.6 & 84.7 & 58.3 & 67.2 & 79.7 & 66.4 & 55.9 & 78.6 & 57.1 & 0 & 0 & 0 & 65.7 & 82.7 & 61.2 \\
\hline Stats: & \multicolumn{3}{|c|}{$p<0.0001$} & \multicolumn{3}{|c|}{$p<0.05$} & \multicolumn{3}{|c|}{ n.s. } & \multicolumn{3}{|c|}{ n.s. } & \multicolumn{3}{|c|}{$p<0.0001$} \\
\hline O Utn $\%$ & 27.4 & 27.4 & 29.8 & 31.5 & 31.6 & 32.3 & 15.4 & 17.4 & 15.4 & 0 & 0 & 0 & 28.3 & 28.7 & 30.0 \\
\hline E Utn \% & 48.2 & 48.2 & 49.6 & 54.0 & 53.8 & 54.8 & 31.6 & 33.3 & 31.6 & 0 & 0 & 0 & 49.7 & 49.9 & 50.8 \\
\hline $\mathrm{E} \mathrm{Fz} \%$ & 48.2 & 48.2 & 49.6 & 54.0 & 53.8 & 54.8 & 31.6 & 33.3 & 31.6 & 0 & 0 & 0 & 49.7 & 49.9 & 50.8 \\
\hline Preg/In \% & 117.4 & 117.4 & 126.3 & 50.0 & 53.3 & 53.8 & 33.3 & 100 & 33.3 & 0 & 0 & 0 & 85.7 & 92.3 & 91.4 \\
\hline Preg/OPU \% & 117.4 & 117.4 & 126.3 & 53.3 & 53.3 & 53.8 & 33.3 & 100 & 33.3 & 0 & 0 & 0 & 85.7 & 92.3 & 91.4 \\
\hline Preg/ET \% & 55.1 & 55.1 & 55.8 & 28.6 & 28.6 & 29.2 & 16.7 & 33.3 & 16.7 & 0 & 0 & 0 & 43.4 & 45.0 & 43.8 \\
\hline EPL \% & 29.6 & 29.6 & 33.3 & 12.5 & 14.3 & 14.3 & 0.0 & 0.0 & 0.0 & 0 & 0 & 0 & 25.0 & 25.0 & 28.1 \\
\hline Stats: & \multicolumn{3}{|c|}{ n.s. } & \multicolumn{3}{|c|}{ n.s. } & \multicolumn{3}{|c|}{ n.s. } & \multicolumn{3}{|c|}{ n.s. } & \multicolumn{3}{|c|}{ n.s. } \\
\hline LB/ET \% & 38.8 & 38.8 & 37.2 & 25.0 & 25.0 & 25.0 & 16.7 & 33.3 & 16.7 & 0 & 0 & 0 & 32.5 & 33.8 & 31.5 \\
\hline Stats: & \multicolumn{3}{|c|}{ n.s. } & \multicolumn{3}{|c|}{ n.s. } & \multicolumn{3}{|c|}{ n.s. } & \multicolumn{3}{|c|}{ n.s. } & \multicolumn{3}{|c|}{ n.s. } \\
\hline LB/Init \% & 82.6 & 82.6 & 84.2 & 43.8 & 46.2 & 46.2 & 33.3 & 100 & 33.3 & 0 & 0 & 0 & 64.3 & 69.2 & 65.7 \\
\hline LB/OPU \% & 82.6 & 82.6 & 84.2 & 43.8 & 46.7 & 46.2 & 33.3 & 100 & 33.3 & 0 & 0 & 0 & 64.3 & 69.2 & 65.7 \\
\hline Stats: & \multicolumn{3}{|c|}{ n.s. } & \multicolumn{3}{|c|}{ n.s. } & \multicolumn{3}{|c|}{ n.s. } & \multicolumn{3}{|c|}{ n.s. } & \multicolumn{3}{|c|}{ n.s. } \\
\hline
\end{tabular}

*Fertilization rate for IVF: denominator adjusted from total 425 oocytes, to 358 oocytes reflecting likely number of MII oocytes (presumed to be the same as ICSI group).

Again, despite the significantly higher fertilization numbers and rates shown in Table 6, the embryo utilization rates (embryos resulting in ETs or cryopreserved) were similar between the IVF-generated and the ICSI-generated. Hence, 
as shown in Table 7, the pregnancy productivity rates (from fresh ETs and FETs), the miscarriage rates (EPLs) and the live birth productivity rates were not significantly different, neither for the total group nor the individual age groupings.

Table 8 Summarizes the raw data outcomes of 26 IVF-ICSI Split Cycles from 26 women who reached OPU and displayed fertilization in one arm only (IVF or ICSI) following random allocation of their oocytes

\begin{tabular}{|c|c|c|}
\hline \multicolumn{3}{|c|}{ IVF-ICSI Split Cycles 2011-2019 Fertilization in one arm only - numbers } \\
\hline & ICSI fertilization only & IVF fertilization only \\
\hline Total initiated cycles & 24 & 2 \\
\hline Women represented & 24 & 2 \\
\hline Age range & 23-42 years & $35-38$ years \\
\hline Cancelled cycles & 0 & 0 \\
\hline OPU procedures & 24 & 2 \\
\hline Oocytes collected (OCCs) & 284 & 14 \\
\hline Oocytes/ OPU (range) & $5-27$ & $5-9$ \\
\hline Oocytes injected (ICSI) & 128 & 6 \\
\hline Oocytes inseminated (IVF)* & $142 / 128$ & $7 / 5$ \\
\hline Total 2PNs (ICSI-derived) & 98 & 0 \\
\hline Total 2PNs (IVF-derived) & 0 & 2 \\
\hline Fresh ET cycles & 20 & 1 \\
\hline Embryos transferred at ET & 22 & 1 \\
\hline Total embryos frozen & 37 & 1 \\
\hline FET cycles & 18 & 1 pending \\
\hline Embryos transferred at FET & 40 & 1 for donation \\
\hline Total embryos utilized & 59 & 2 \\
\hline Fresh ET pregnancies & 5 & 0 \\
\hline FET pregnancies & 9 & Pending\# \\
\hline Total Fresh \& Fz pregs & 14 & $0+1$ pending\# \\
\hline EPLs & 2 & 0 \\
\hline Fresh ET live births & 5 & Nil? \\
\hline FET Live births & 7 & Nil? \\
\hline Total fresh \& Fz live births & 12 & Nil? \\
\hline
\end{tabular}

*Denotes 142 OCCs/ assumes 128 at MII stage (as determined following cumulus stripping for ICSI; similar for 7/5. \#One woman had freeze-all (deferring ET for pending myomectomy); high quality blastocyst (5BA by Gardner grading) frozen, now offered for donation.

Table 8 displays the laboratory and clinical outcomes from 26 women in both young and older age ranges who demonstrated fertilization in one arm only of their randomized oocyte allocation. Fertilization in only the ICSI arm occurred in 24 occasion (from 24 women) where the OPU range was 5-27 OCCs. Those women generated 98 embryos from 128 oocytes injected by ICSI (76.6\% fertilization rate) but nil from 142 OCCs exposed to IVF (0/128 adjusted fertilization rate). Fertilization in only the IVF arm occurred on 2 occasions (from 2 women) where the OPU range was 5-9 OCCs. Those women generated only 2 embryos from 7 oocytes inseminated by IVF (28.6\%, adjusted to $40.0 \%$ fertilization) but nil from 6 oocytes MII oocytes treated by ICSI ( $0.0 \%$ fertilization). 
Table 9 The rates and clinical outcomes of 26 IVF-ICSI Split Cycles from 26 women who reached OPU and displayed fertilization in one arm only (IVF or ICSI) following random allocation of their oocytes

\begin{tabular}{|c|c|c|}
\hline \multicolumn{3}{|c|}{ IVF-ICSI Split Cycles 2011-2019 Fertilization in one arm only - rates (\%) } \\
\hline & ICSI fertilization only & IVF fertilization only \\
\hline Total initiated cycles & 24 & 2 \\
\hline Women represented & 24 & 2 \\
\hline Age range & $23-42$ years & 35-38 years \\
\hline Cancellation rate $\%$ & 0.0 & 0.0 \\
\hline OPU procedures & 24 & 2 \\
\hline Oocytes collected & 284 & 14 \\
\hline Oocytes/ OPU mean (range) & $11.8(5-27)$ & $7.0(5-9)$ \\
\hline Oocytes injected (ICSI) & 128 & 5 \\
\hline Oocytes inseminated (IVF)* & $142 / 128$ & $7 / 5$ \\
\hline Fertilization rate (ICSI) \% & 76.6 & 0.0 \\
\hline Fertilization rate (IVF) \% & 0.0 & 40.0 \\
\hline Oocyte utilization \% & 20.8 & 14.3 \\
\hline Embryo utilization \% & 60.2 & 100 \\
\hline Pregnancy rate Fresh ET & 20.8 & 0.0 \\
\hline Pregnancy rate FET & 50.0 & Not utilized\# \\
\hline Live birth rate Fresh ET & 20.8 & 0.0 \\
\hline Live birth rate FET & 38.9 & 5BA embryo not utilized\# \\
\hline EPLs & 14.3 & No pregnancies \\
\hline PPR / Initiated cycle & 58.3 & Nil? \\
\hline PPR/ OPU & 58.3 & Nil? \\
\hline PPR/ET & 70.0 & Nil? \\
\hline LBP/Initiated cycle & 50.0 & Nil? \\
\hline $\mathrm{LBP} / \mathrm{OPU}$ & 50.0 & Nil? \\
\hline $\mathrm{LBP} / \mathrm{ET}$ & 60.0 & Nil? \\
\hline
\end{tabular}

*Denotes 142 OCCs/ assumes 128 at MII stage (as determined following cumulus stripping for ICSI; similar for 7/5. \#0ne woman had freeze-al (deferring ET for pending myomectomy); high quality blastocyst (5BA by Gardner grading) frozen, now offered for donation.

The embryo utilization and clinical outcomes are shown in Table 9 where it can be seen that both oocyte and embryo utilization rates were comparable for both IVF and ICSI, but pregnancies only occurred in the ICSI group where live birth productivity outcome was $50.0 \%$ per initiated cycle. However, one of the 2 women in the IVF arm had her sole good-quality blastocyst embryo cryopreserved, pending a myomectomy procedure. However, this has not eventuated during the study period and the woman has offered the embryo for anonymous donation. If that embryo was given thew opportunity for FET, the possibility is that the potential live birth ratio could have been $12 / 24$ (50\%) for ICSI and 1 from $2(50 \%)$ for IVF (no statistical difference). 
Table 10 Summarizes the raw data and rates for the clinical outcomes from a further 86 FET cycles following the first ET or FET treatment cycle from the original 242 IVF-ICSI Split cycles

\begin{tabular}{|c|c|c|c|}
\hline & ICSI-generated & IVF-generated & Total \\
\hline Cycles with FET & 38 & 44 & 86 \\
\hline \multirow[t]{2}{*}{ Pregnancies n, \% per ET } & $2463.2 \%$ & $818.2 \%$ & 32 \\
\hline & \multicolumn{2}{|c|}{$\mathrm{p}<0.0001$} & \\
\hline EPLs n, \% & $312.5 \%$ & $225.0 \%$ & 5 \\
\hline Live births n, \% per ET & $2155.3 \%$ & $613.6 \%$ & 27 \\
\hline \multirow[t]{2}{*}{ Babies } & 22 & 6 & 28 \\
\hline & \multicolumn{2}{|c|}{$\mathrm{p}<0.0001$} & \\
\hline
\end{tabular}

The 86 FET cycles shown in Table 10 denote secondary FET procedures following the first round ET plus FET from the 242 treatment cycles shown in Tables 2 . The women had failure to achieve pregnancy in the first round or did achieve a pregnancy with either miscarriage or live birth outcomes. The main observation is that the pool of cryopreserved embryos from which this data is drawn is a secondary group, where the best quality embryos have already been sourced for the ET and FET treatments undertaken in the first round. It can be seen that 24 pregnancies arose from 38 FET cycles using ICSI-generated embryos and only 8 pregnancies arose from 44 FET cycles using IVF-generated embryos. The live birth rates were also significantly different being $55.3 \%$ for ICSI-generated embryos and $13.6 \%$ for IVF-generated embryos $(\mathrm{p}<0.0001)$.

Table 11 Summarizes the raw data and rates for the clinical outcomes from those embryos remaining in cryopreservation from the original 242 IVF-ICSI Split cycles. The potential outcomes are estimated from the actual rates arising in Table 3

\begin{tabular}{|c|c|c|}
\hline \multicolumn{2}{|c|}{242 Autologous IVF-ICSI Split Cycles - potential from remaining Fz embryos } \\
\hline & ICSI-generated & IVF-generated \\
\hline Total embryos in cryostorage & 302 blastocysts & 43 blastocysts \\
\hline Age $<35$ years & 204 & 39 \\
\hline - $\quad$ Pregs/ET 42.6\%, 56.8\%* & 88 pregnancies & 34 pregnancies \\
\hline - LBR/ET 37.5\%, 42.3\%* & 77 babies & 17 babies \\
\hline Age $\geq 35$ years & 98 & 4 \\
\hline - Pregs/ET 44.9\%, 33.8\%* & 44 pregnancies & 2 pregnancies \\
\hline - LBR/ET 31.9\%, 24.6\%* & 32 babies & 1 baby \\
\hline All women; potential pregnancies & 132 pregnancies & 36 pregnancies \\
\hline All women; potential births & 109 births & 18 births \\
\hline All women; potential babies & 110 babies & 18 babies \\
\hline \multicolumn{2}{|c|}{$*$ Rates derived from Table 3. \#Includes 1 monozygotic twinning } \\
\hline
\end{tabular}

By completion of the study period, January 2011 to December 2019, there were 345 blastocyst embryos remaining in cryostorage and which had been generated from the 233 women who had 242 IVF-ICSI Split treatment cycles. Table 11 shows the distribution of those embryos according to the women's age grouping and the derivation of the embryos, being ICSI-generated $(n=302)$ or IVF-generated $(n=43)$. The marked difference in embryo numbers between ICSI and IVF related to the highly significant fertilization rates, shown in Table 2 and Table 3 . By utilization the pregnancy and live birth rates shown in Table 3, the potential clinical outcomes for those remaining embryos in cryostorage, has been 
applied to project the likely potential livebirths in Table 11. There is likely to be 109 live births from ICSI and only 18 births from IVF, a significant difference for the 233 women having ART procedures $(\mathrm{p}<0.0001)$.

Table 12 Summarizes the raw data for the actual and potential clinical outcomes arising from the original 242 IVF-ICSI Split cycles and includes the total productivity from fresh ETs, the first FET treatment cycle, any subsequent FETS in the timeframe (2011-2019) as well as the potential productivity (pregnancies, live births and babies) from those embryos remaining in cryostorage. The statistical analysis is shown in Figure 2

\begin{tabular}{|c|c|c|c|}
\hline \multicolumn{4}{|c|}{242 Autologous IVF-ICSI Split Cycles - total productivity } \\
\hline 199 Cycles with fresh ET & ICSI-generated & IVF-generated & Totals \\
\hline - $\quad$ Live births from ET* & 36 births* & 34 births* & 70 births* \\
\hline - $\quad$ Babies from ET & 37 babies & 34 babies & 71 babies \\
\hline - $\quad$ Live births from FET & 34 births & 33 births & 67 births \\
\hline - $\quad$ Babies from FET & 35 babies & 34 babies & 69 babies \\
\hline - $\quad$ Live births from ET + FET & 70 births & 67 births & 137 births \\
\hline - $\quad$ Babies from ET + FET & 72 babies & 68 babies & 140 babies \\
\hline \multicolumn{4}{|l|}{42 Freeze-all Cycles } \\
\hline - $\quad$ Live births, all FET & 14 births & 13 births & 27 births \\
\hline - $\quad$ Babies from FET & 15 babies & 14 babies & 29 babies \\
\hline \multicolumn{4}{|l|}{242 Cycles - First pregnancy } \\
\hline - $\quad$ Live births from ET \& FET & 84 births & 80 births & 164 births \\
\hline - $\quad$ Babies & 87 babies & 83 babies & 170 babies \\
\hline \multicolumn{4}{|l|}{242 Cycles + Subsequent pregs } \\
\hline - $\quad$ Live births from FET & $84+21=$ births & $80+6$ births & 191 births \\
\hline - $\quad$ Babies & $87+22$ babies & $83+6$ babies & 198 babies \\
\hline Potential Cycles from Cryostorage & 132 pregnancies & 36 pregnancies & \\
\hline + Potential births from FET & 109 births & 18 births & 127 births \\
\hline Potential babies from FET & 110 babies & 18 babies & 128 births \\
\hline All live births (actual + potential) & $84+21+109$ & $80+6+18$ & 318 births \\
\hline Total babies (actual + potential) & $109+110$ babies & $89+18$ babies & 326 babies \\
\hline
\end{tabular}

The 2 singleton live births arising have been allocated according to the highest quality grading of the embryos, one by ICSI and one by IVF. No congenital abnormalities were detected in any of the babies (neither IVF nor ICSI generated).

Compiling the live birth data from the various IVF-ICSI treatment groups shown in Table 2, Table 4, Table 6 and Table 8 enables a calculation of actual live births and babies born from this study. By adding in the potential data show in Table 10 enables a complete picture showing that 318 births generated 326 babies (Table 12) from 233 women who undertook 242 treatment cycles. This is shown in graphical form in Figure 1. 
Figure 2 Flowsheet documenting the clinical outcomes indicating the derivation of live births and babies delivered arising from the primary data set of the 242 IVF-ICSI Split treatment cycles and the derivation of those outcomes from either IVF or ICSI generated embryos. The data includes the total productivity from fresh ETs, the first FET treatment cycle, any subsequent FETs in the timeframe (2011-2019) as well as the potential productivity (pregnancies, live births, and babies) from those embryos remaining in cryostorage. The statistical analysis compares the birth rates of ICSIderived vs IVF-derived pregnancies.

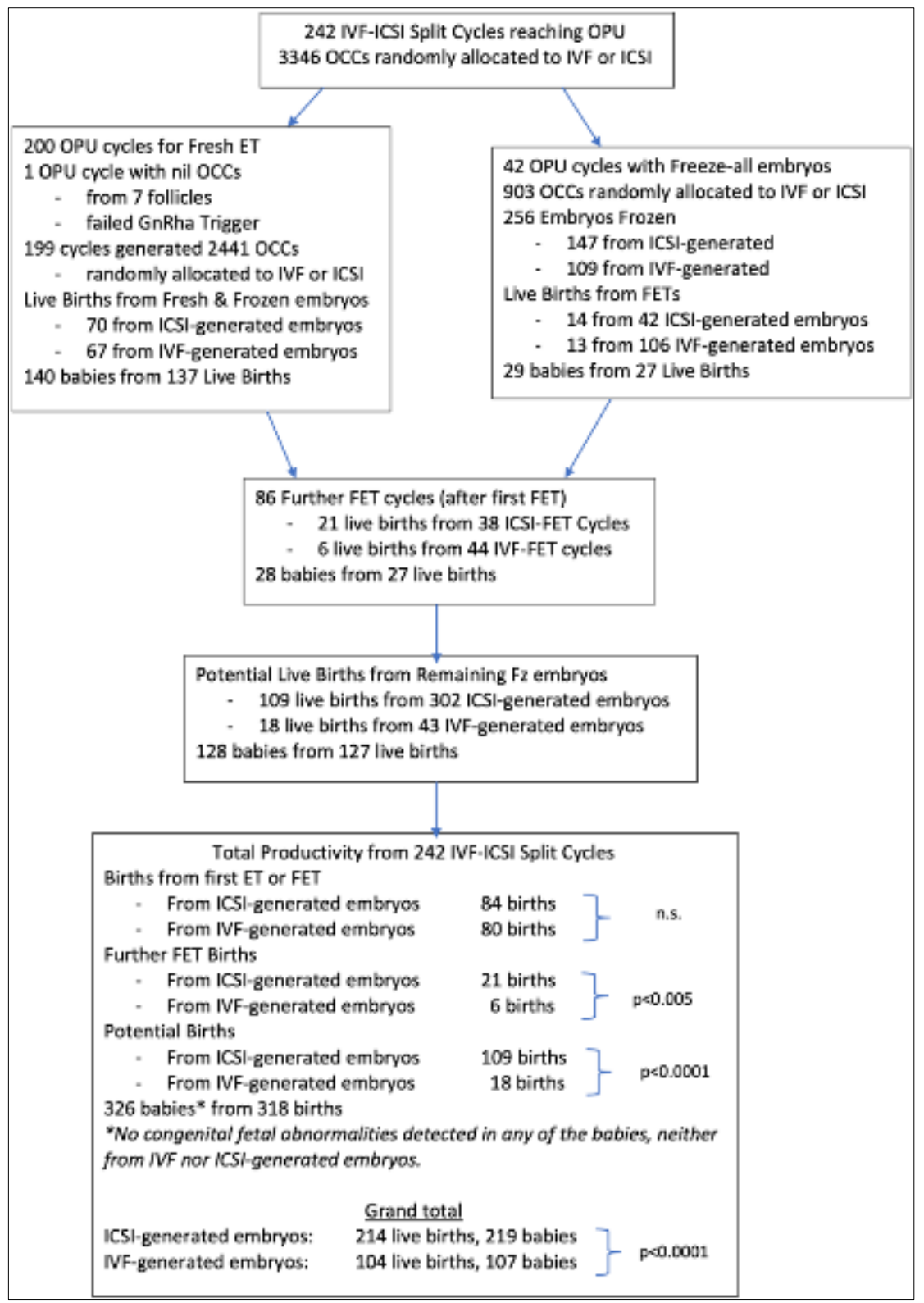

\section{Discussion}

The data presented in this study examines an IVF-ICSI Split model for couples with unexplained infertility, where male factor is meticulously excluded and ART is conducted by a strict algorithm, a commitment to blastocyst culture, along with single embryo transfers and a high commitment to cryopreservation. From 242 treatment cycles, 3346 oocytes recovered (13.8 per OPU) were randomly allocated to IVF or ICSI and the fertilization rates standardized to the number of 2PNS arising from each group applying the metaphase II oocyte number identified for the ICSI group, as the denominator for both groups. Applying this adjusted formula for fertilization by IVF, we found the fertilization rates were significantly higher overall for ICSI (83.2\% vs 65.4\%; $\mathrm{p}<0.0001)$, being most pronounced for women under 40 years. 
Nonetheless, we found that the resultant embryos from either IVF or ICSI had equivalent utilization rates for both fresh ETs and FETs so that pregnancy rates and ensuing live birth rates were equivalent in the first round of procedures. However, pursuant to the higher fertilization rates for ICSI, there were significantly higher numbers of ICSI-generated embryos cryopreserved and subsequent FET procedures (after the first round) showed higher live birth rates for the ICSI-generated embryos. This likely reflects the more diminished pool of embryos in cryostorage from the IVF arm, with the remainder comprising a smaller pool of lower quality embryos from which to select for second and subsequent FET procedures. On the contrary, the ICSI-generated pool of cryostored embryos comprised a significantly larger pool, with undoubtedly many high-quality embryos from which to select for further FET procedures.

Furthermore, our calculation of the potential live births from those embryos remaining in cryostorage at the completion of this study, indicates a further 214 live births potentially arising from the ICSI-generated embryos as opposed to 104 live births from the IVF-generated embryos. Our data is further reassuring as all the babies delivered from the live births in this study $(198$ babies comprising 89 babies from 86 IVF-generated births and 109 babies from 105 ICSI-generated births) were all healthy with no congenital abnormalities detected. This data might support the idea of encouraging ICSI fertilization for all ART cases, however we did show that a small proportion of women will demonstrate fertilization in the IVF-arm only (7.7\%) without a single oocyte fertilizing from 5 injected in that same group. For this reason, PIVET encourages and practices a 90\% ICSI fertilization rate and maintains the IVF-ICSI Split model for all IVF-naïve women categorized with unexplained infertility and where male factor has been clearly excluded following investigations which include a thorough clinical assessment, male urogenital ultrasound examination, semen profiling, ASAB and DNA fragmentation screening. The results from the IVF-ICSI mode carries high diagnostic predictive value and provides the lead for future ART procedures when required.

Although the study has a definable weakness in being a retrospective analysis of the IVF-ICSI Split mode of fertilization, it also has a strong positive feature in that the OCCs were allocated randomly by the embryologists, without reference to the various Consultant Clinicians who, although closely managing the procedural side of the women's management, were completely functioning outside the laboratory aspects which were decided by the embryology team, under the general direction of the Medical Director and the Laboratory Director.

Whilst the data from ICSI at PIVET clearly favors ICSI, this has not been a universal viewpoint [72,73] and the ASRM Practice committee has repeatedly advised against ICSI for non-male factor infertility [73,74]. Nonetheless, we have argued that our studies have applied a specific approach utilizing clear clinical criteria which includes a favorable FSHdosing algorithm [7,45], a single embryo transfer policy of almost $100 \%$, a blastocyst culture protocol for $>90 \%$ of couples [45] and an ICSI protocol with injection of oocytes strictly $42 \pm 2$ hours post HCG Trigger [68]. Furthermore, we have evaluated the male partner most carefully [75], with attention to clinical features as well as testing for antispermatozoal antibodies [8,76], DNA-fragmentation [40] and research with the hypo-osmotic swelling test, with the view to identifying the individual sperm with minimal DNA fragmentation [54]. We are aware that such intensive assessment of the male partner is not universally practiced [75]. However, we are aware that our protocols place our PIVET clinic in the highest quartile for live birth outcomes from initiated cycles in the Australian and New Zealand Assisted Reproduction Database [52]. It also means that our IVF \pm ICSI program removes any relevance of male age or semen parameters on clinical pregnancy and live birth outcomes [77]. Hence, we have no reservations about our data not being concordant with the past mainstream. We would respectfully suggest that other facilities trial PIVET protocols.

\section{Conclusion}

This study corroborates our view that most (90\%) ART procedures should be conducted using the ICSI technique for fertilization - both for male factor and most non-male factor cases. However, the decision for using ICSI on cases of unexplained infertility should be decided by undertaking an IVF-ICSI Split method on all IVF-naïve women. This will enable the detection of those cases best suited to ICSI as well as avoiding cases of complete failed fertilization. It will also ensure that every couple has a reasonable chance of generating a live birth on their first ART treatment cycle.

\section{Compliance with ethical standards}

\section{Acknowledgments}

We are grateful for the close working relationship between the various Departments operating at PIVET Medical Centre, particularly the integration of nursing, laboratory and clinical areas for the daily Results Review meeting and the handling of patients within the Perth Day Surgery Centre for OPU and ET procedures. The nursing team at PIVET have 
been fastidious in detailing clinical outcomes into registers, thereafter into the Filemaker database. Nurse Alison Pusey has been especially successful in tracking the outcome of each pregnancy, many of which resulted in deliveries in regional locations and sometimes overseas.

\section{Disclosure of conflict of interest}

The entire project has been funded internally at PIVET without any external or commercial contributions. The authors declare no conflicts of interest.

\section{Statement of ethical approval}

Reporting of the data was approved under Curtin University Human Ethics Committee approval no. RD-25-10 general approval for retrospective data analysis in 2010, updated in 2015, and again further updated in August 2020.

\section{Statement of informed consent}

PIVET is accredited with both the self-regulatory National Australian Reproductive Technology Committee (RTAC) as well as the Reproductive Technology Council (RTC) of Western Australia. Consent forms received approval under both regulatory bodies. The assay laboratory is accredited on an annual basis by the National Australian Testing Authority (NATA).

\section{Author Contributions}

The study was conceived by PIVET Medical Director JLY who established the data base at PIVET Medical Centre with the assistance of IT Consultant and data manager PMH. Lab Director JLC has directed embryologists NM, RG and JW in oocyte allocation for the project. All authors have assisted with the data analyses as well as the preparation of the Tables and Figures. The manuscript was written by JLY and each of the authors has read and agreed to its content.

\section{References}

[1] Edwards R, Bavister B, Steptoe, P. (1969). Early stages of fertilization In vitro of human oocytes matured In vitro. Nature, 221, 632-635.

[2] Steptoe PC, Edwards RG. (1978). Birth after the re-implantation of a human embryo. Lancet, 2(8085), 366.

[3] Yovich JL, Craft IL. (2018). Founding pioneers of IVF: Independent innovative researchers generating livebirths within 4 years of the first birth. Reprod Biol, 18, 317-323.

[4] Yovich JL. (2020). Founding pioneers of IVF Update: Independent innovative researchers generating livebirths within 4 years of the first birth. Reprod Biol, 20, 111-113.

[5] Craft I, Yovich J. (1979). Implications of embryo transfer. Lancet, ii, 642-643.

[6] Yovich JL, Matson PL. (1989). The influence of infertility aetiology on the outcome of in-vitro fertilization (IVF) and gamete intrafallopian transfer (GIFT) treatments. Int J Fertil, 35, 26-33.

[7] Yovich JL. (2019). Indications and Techniques of In vitro fertilization. In: 40 years after In vitro fertilization: state of the art and new challenges (ed: Jan Tesarik). Cambridge Scholars Publishing, Lady Stephenson Library, Newcastle upon Tyne, UK, 25-75.

[8] Yovich JL, Stanger JD, Kay D, Boettcher B. (1984). In-vitro fertilization of oocytes from women with serum antisperm antibodies. Lancet, ii, 369-370.

[9] Yovich JL, Stanger JD. (1984). The limitations of in-vitro fertilization from males with severe oligospermia and abnormal sperm morphology. J In vitro Fert Embryo Transfer, 1(3), 172-179.

[10] Mahadevan M, Baker G. (1984). Assessment and preparation of semen for In vitro fertilization. In C. Wood et al (eds). Clinical In vitro Fertilization. Springer-Verlag Berlin Heidelberg, Ch7, 83-97.

[11] Yovich JL, Stanger JD, Yovich JM. (1985). Management of oligospermic infertility by in-vitro fertilization. In: In vitro Fertilization and Embryo Transfer. Seppälä M, Edwards RG. (Eds). Ann NY Acad Sci, 442, 276-286.

[12] Yovich JM, Edirisinghe WR, Cummins JM, Yovich JL. (1988). Preliminary results using pentoxifylline in a PROST program for severe male factor infertility. Fertil Steril, 50, 179-181. 
[13] Yovich JM, Edirisinghe WR, Cummins JM, Yovich JL. (1990). Influence of pentoxifylline in severe male factor infertility. Fertil Steril, 53(4), 715-722.

[14] Yovich JL. (1993). Pentoxifylline: actions and applications in assisted reproduction. Hum Reprod, 8, $1786-91$.

[15] Malter HE, Cohen J. (1989). Partial zona dissection of the human oocyte: a non-traumatic method using micromanipulation to assist zona pellucida penetration. Fertil Steril, 51(1), 139-148.

[16] Ng SC, Bongso A, Sathananthan H, Chan CL, Wong PC, Hagglund L, Ratnam SS. (1988). Pregnancy after transfer of sperm under zona. Lancet, 2: 790.

[17] Edirisinghe WR, Junk SM, Yovich JM, Bootsma B, Yovich JL. (1995). Sperm stimulants can improve fertilization rates in male-factor cases undergoing IVF to the same extent as micromanipulation by partial zona dissection (PZD) or sub-zonal sperm insemination (SUZI): a randomized controlled study. J Assist Reprod Genet, 12(5), 312318.

[18] Ng SC, Bongso TA, Liow SL, Edirisinghe R, Ratnam SS. (1992). Micro-insemination sperm transfer (MIST) and its application to male subfertility: current strategies to improve results. Ann Acad Med Singa, 21(4), 561-564.

[19] Palermo G, Joris H, Devroey P, Van Steirteghem AC. (1992). Pregnancies after intracytoplasmic injection of single spermatozoon into an oocyte. Lancet, $340(8810), 17-18$.

[20] Yovich JL, Edirisinghe WR, Apted SI, Yovich JM. (1993). Pregnancy from microinjected epididymal spermatozoa. Med J Aust, 159, 71-72.

[21] Yovich JL. (1993). Individualization of sperm preparations. J Assist Reprod Genet, 10, 247-250.

[22] Matson PL, Yovich JM, Edirisinghe WR, Junk SM, Yovich JL. (1995). An argument for the past and continued use of pentoxifylline in assisted reproductive technology. Hum Reprod, 10, suppl 1, 67-71.

[23] Molina LCP, Luque GM, Balestrini PA, Marin-Briggiler, CI, Romarowski A, Buffone MG. (2018). Molecular basis of human sperm capacitation. Front Cell Dev Biol, 6, 1-72.

[24] Holt WV, Fazeli A. (2015). Do sperm possess a molecular passport? Mechanistic insights into sperm selection in the female reproductive tract. Molecular Hum Reprod, 21, 491-501.

[25] De Jonge C. (2005). Biological basis for human capacitation. Hum Reprod Update, 2005, 11(3), $205-214$.

[26] De Jonge C. (2017). Biological basis for human capacitation - revisited. Hum Reprod Update, 23(3), $289-299$.

[27] Suarez SS, Ho H-C. (2003). Hyperactivation of mammalian sperm. Cell Mol Biol, 49(3), 351-356.

[28] Yovich JM, Edirisinghe WR, Yovich JL. (1994). Use of the acrosome reaction to ionophore challenge test (ARIC) in managing patients in an assisted reproduction programme. Fertil Steril, 61, 902-910.

[29] Sosa CM, Pavarotti MA, Zanetti MN, Zoppino FCM, De Blas GA, Mayorga LS. (2015). Kinetics of human sperm acrosomal exocytosis. Mol Hum Reprod, 21(3), 244-254.

[30] Flesch FM, Gadella BM. (2000). Dynamics of the mammalian sperm plasma membrane in the process of fertilization. Biochimica et Biophysica Acta, 1469, 197-235.

[31] Gadella B. (2012). Dynamic regulation of sperm interactions with the zona pellucida prior to and after fertilisation. Reprod Fert Develop, 25(1), 26-37.

[32] Tosti E, Menezo Y. (2016). Gamete activation: basic knowledge and clinical applications. Hum Reprod Update, 22(4), 420-439.

[33] Yeste M, Jones C, Amdani SN, Patel S, Coward K. (2016). Oocyte activation deficiency: a role for an oocyte contribution? Hum Reprod Update, 22(1), 23-47.

[34] Kashir J, Nomikos M, Swann K, Lai FA. (2015). PLC-zeta or PAWP: revisiting the putative mammalian sperm factor that triggers egg activation and embryogenesis. Mol Hum Reprod, 21(5), 383-388.

[35] Simerly CR, Takahashi D, Jacoby E, Castro C, Hartnett C, Hewitson L, Navara C, Shatten G. (2019). Fertilization and cleavage axis differ in primates conceived by conventional (IVF) versus intracytoplasmic sperm injection (ICSI). Sci Rep, 9, 15282.

[36] Avidor-Reiss T, Mazur M, Fishman EL, Sindhwani P. (2019). The role of sperm centrioles in human reproduction - the known and the unknown. Front Cell Dev Biol, 7(188), 1-15. 
[37] Sanchez AD, Feldman JL. (2017). Microtubule-organising centers: from the centrosome to non-centrosomal sites. Current Opinion in Cell Biology, 44, 93-101.

[38] Jaroudi K, Al-Hassan S, Al-Sufayan H, Al-Mayman H, Qeba M, Coskun S. (2003). Intracytoplasmic sperm injection and conventional In vitro fertilization are complementary techniques in management of unexplained infertility. J Assist Reprod Genet, 20(9), 377-381.

[39] Vitek WS, Galarraga O, Klatsky PC, Robins JC, Carson SA, Blazar AS. (2013). Management of the first In vitro fertilization cycle for unexplained infertility: a cost-effectiveness analysis of split In vitro fertilizationintracytoplasmic sperm injection. Fertil Steril, 100(5), 1381-1388.

[40] Yovich JL, Mustafa KB, Marjanovich N, Yovich SJ, Keane KN. (2016). IVF-ICSI Split insemination reveals those cases of unexplained infertility benefitting from ICSI even when the DNA fragmentation index is reduced to $15 \%$ or even 5\%. Androl Gynecol: Curr Res, 4(1), 1-7.

[41] Yovich JL, Conceicao JL, Marjanovich N, Ye Y, Hinchliffe PM, Dhaliwal SS, Keane KN. (2018). An ICSI rate of 90\% minimizes complete failed fertilization and provides satisfactory implantation rates without elevating fetal abnormalities. Reprod Biol, 18, 301-311.

[42] Sauerbrun-Cutler M-T, Huber WJ 3rd, Has P, Shen C, Hackett, Alvero R, Wang S. (2020). Is intracytoplasmic sperm (ICSI) better than traditional In vitro fertilization (IVF): confirmation of higher blastocyst rates per oocyte using a split insemination design? J Assist Reprod Genet, 37(7), 1661-1667.

[43] Yovich JL, Conceicao JL, Wong J, Marjanovich N, Wicks R, Hinchliffe PM. (2021). Fertilization by ICSI generates a higher number of live births than IVF in a pioneer facility applying $>90 \%$ single blastocyst-stage embryo transfers. GSC Biological and Pharmaceutical Sciences, 15(01), 087-103.

[44] Yovich JL, Puzey A, De Atta R, Roberts R, Reid S, Grauaug A. (1982). In-vitro fertilization pregnancy with early progestagen support. Lancet, ii, 378-379.

[45] Yovich JL. (2019). Monitoring the stimulated IVF cycle. In: How to Prepare the Egg and Embryo to Maximise IVF Success. (Eds: Gabor T Kovacs, Anthony J Rutherford, David K Gardner). Cambridge University Press, Cambridge, 94-120.

[46] Yovich J, Stanger J, Hinchliffe P. (2012). Targeted gonadotrophin stimulation using the PIVET algorithm markedly reduces the risk of OHSS. Reprod Biomed Online, 24(3), 281-292.

[47] Yovich JL, Alsbjerg B, Conceicao JL, Hinchliffe PM, Keane KN. (2016). PIVET rFSH dosing algorithms for individualized controlled ovarian stimulation enables optimized pregnancy productivity rates and avoidance of ovarian hyperstimulation syndrome. Drug Des Devel Ther, 10, 2561-2573.

[48] Yovich JL, Keane KN, Borude G, Dhaliwal SS, Hinchliffe PM. (2018). Finding a place for corifollitropin within the PIVET FSH-dosing algorithms. Reprod Biomed Online, 36(1), 47-58.

[49] Yovich JL, Hinchliffe PM, Lingam S, Srinivasan S, Keane KN. (2018). Adjusting the PIVET rFSH dosing algorithm for the biosimilar Bemfola product. J Fertil In vitro IVF Worldw Reprod Med Genet Stem Cell Biol, 5: 3.

[50] Kuwayama M, Vajta G, Kato O, Leibo SP. (2005). Highly efficient vitrification method for cryopreservation of human oocytes. Reprod Biomed Online, 11, 300-308.

[51] Newman JE, Paul RC, Chambers GM. (2020). Assisted Reproductive Technology in Australia and New Zealand 2018; National Perinatal Epidemiology and Statistics Unit, the University of New South Wales: Sydney, Australia, 1-83.

[52] Your IVF Success Estimator. (2020). Find an Australian IVF clinic. Australian \& New Zealand Assisted Reproduction Database. University of New South Wales: Sydney, Australia.

[53] WHO. (2010). WHO laboratory manual for the examination and processing of human semen, 5th ed. Geneva: World Health Organization, 270pp.

[54] Stanger JD, Vo L, Yovich JL, Almahbobi G. (2010). Hypo-osmotic swelling test identifies individual spermatozoa with minimal DNA fragmentation. Reprod Biomed Online, 20, 474-484.

[55] Yovich JL, Katz D, Jequier AM. (2018). Sperm recovery for men with spinal cord injury: vasal flush is the preferred method for an-ejaculatory males. J Fertil In vitro IVF Worldw Reprod Med Genet Stem Cell Biol, 6(1), 1-6.

[56] Jequier AM. (2011). Physical agents, toxins and drugs: their effect upon male fertility. In: Male Infertility. A Clinical Guide. Cambridge University Press. Second Edition, 197-208. 
[57] Yovich JL, Hinchliffe PM. (2021). A 10-year perspective on the utility of three adjuvants often used in IVF: growth hormone, melatonin and DHEA. Reprod Med, 2, 155-162.

[58] Boulet SL, Mehta A, Kissin DM, Warner L, Kawwass JF, Jamieson DJ. (2015). Trends in use of and reproductive outcomes associated with intracytoplasmic sperm injection. JAMA, 313(3), 255-63.

[59] Ludwig M, al-Hasani S, Küpker W, Bauer O, Diedrich K. (1997). A new indication for an intracytoplasmic sperm injection procedure outside the cases of severe male factor infertility. Eur J Obstet Gynecol Reprod Biol, 75(2), 207-210.

[60] Ou YC, Lan KC, Huang FJ, Kung FT, Lan TH, Chang SY. (2010). Comparison of In vitro fertilization versus intracytoplasmic sperm injection in extremely low oocyte retrieval cycles. Fertil Steril, 93, 96-100.

[61] Gozlan I, Dor A, Farber B, Meirow D, Feinstein S, Levron J. (2007). Comparing intracytoplasmic sperm injection and In vitro fertilization in patients with single oocyte retrieval. Fertil Steril, 87, 515-518.

[62] Zhao J, Zhang N-Y, Xu Z-P, Chen L-J, Zhao X, Zeng H-M, Jiang Y-Q, Sun H-X. (2015). Effects of abnormal zona pellucida on fertilization and pregnancy in IVF/ICSI-ET. J Reprod Contraception, 26, 73-80.

[63] O’Neill CL, Chow S, Rosenwaks Z, Palermo GD. (2018). Development of ICSI. Reproduction, 156, F51-F58.

[64] Van der Westerlaken L, Helmerhorst F, Dieben S, Naaktgeboren N. (2005). Intracytoplasmic sperm injection as a treatment for unexplained total fertilization failure or low fertilization after conventional In vitro fertilization. Fertil Steril, 83, 612-617.

[65] Vitek WS, Galarraga O, Klatsky PC, Robins JC, Carson SA, Blazar AS. (2013). Management of the first In vitro fertilization cycle for unexplained infertility: a cost-effectiveness analysis of split In vitro fertilizationintracytoplasmic sperm injection. Fertil Steril, 100(5), 1381-1388.

[66] Ruiz A, Remohi J, Minguez Y, Guanes PP, Simon C, Pellicer A. (1997). The role of In vitro fertilization and intracytoplasmic sperm injection in couples with unexplained infertility after failed intrauterine insemination. Fertil Steril, 68(1), 171-173.

[67] Yovich JL, Grudzinskas G. (1990). In vitro fertilization and embryo transfer (IVF-ET): current status (1988). In: The Management of Infertility, a manual of gamete handling procedures, Chapter 10. Heinemann Medical Books, Oxford. UK (ISBN 043300160 7), 121-44.

[68] Yovich JL. (2020). The effect of ICSI-related procedural timings and operators on the outcome. GSC Biological and Pharmaceutical Sciences, 11(01), 09-11.

[69] Larbuisson A, Raick D, Demelenne S, Delvigne A. (2017). ICSI diagnostic: a way to prevent total fertilization failure after 4 unsuccessful IUI. Basic Clin Androl, 27(18), 1-5.

[70] Harton GL, De Rycke M, Fiorentino F, Moutou C, SenGupta S, Traeger- Synodinos J, Harper JC. (2011). European Society for Human Reproduction and Embryology (ESHRE) PGD Consortium. ESHRE PGD consortium best practice guidelines for amplification based PGD. Hum Reprod, 26(1), 33-40.

[71] Yovich JL, Mariappen U, Hinchliffe PM, Dhaliwal SS, Keane KN. (2020). MPA given orally during the first trimester for threatened miscarriage carries no specific risk for foetal abnormalities albeit the rate is higher than nonthreatened pregnancies. Reprod Biol, 20, 424-432.

[72] Li Z, Wan AY, Bowman M, Hammarberg K, Farquar C, Johnson L, Safi N, Sullivan EA. (2018). ICSI does not increase the cumulative live birth rate in non-male factor infertility. Hum Reprod, 33(7), 1322-1330.

[73] ASRM Practice Committee. (2012). Intracytoplasmic sperm injection (ICSI) for non-male factor infertility: a committee opinion. Fertil Steril, 98, 1395-1399.

[74] American Society for Reproductive Medicine. (2020). Practice Committee for the Society of Assisted Reproductive technology. Intracytoplasmic sperm injection (ICSI) for non-male factor indications: a committee opinion. Fertil Steril, 114, 239-245.

[75] Yovich JL, Keane KN. (2017). Assessing the male in fertility clinics - men undervalued, under-managed and undertreated. Trans Androl Urol, 6 (suppl 4), s624-s628.

[76] Matson PL, Junk SM, Spittle JW, Yovich JL. (1998). Effect of antispermatozoal antibodies in seminal plasma upon spermatozoal function. International Journal of Andrology, 11(2), 101-106.

[77] Mariappen U, Keane KN, Hinchliffe PM, Dhaliwal SS, Yovich JL. (2018). Neither male age nor semen parameters influence clinical pregnancy or live birth outcomes from IVF. Reprod Biol, 18, 324-329. 


\section{Author's Short Biography}

\begin{tabular}{|l|l|} 
Professor John Yovich MD PhD FRCOG FRANZCOG CREI graduated MBBS, now MD, \\
from the University of Western Australia in 1970, progressing into Specialist O\&G \\
practice in 1976. Following laboratory research and clinical work over 4-years in \\
London 1976-80 with Professor Ian Craft, John presented his MD, now PhD, \\
thesis "Human pregnancies achieved by in-vitro fertilization" (UWA 1985). Established \\
PIVET Medical Centre in 1981, generating WA's first IVF child in July 1982. Assisted \\
many IVF clinics to establish worldwide, forming PIVET Malaysia in Kuala Lumpur with \\
first IVF infant born May 1987, also assisted the first IVF infant for Greece delivered in \\
May 1987 and established Cairns Fertility Clinic in 2008.
\end{tabular}

